\title{
WP 40_10
}

\author{
Miana Plesca \\ Department of Economics, University of Guelph \\ The Rimini Centre for Economic Analysis (RCEA)
}

\section{A GENERAL EQUILIBRIUM EVALUATION OF THE EMPLOYMENT SERVICE}

Copyright belongs to the author. Small sections of the text, not exceeding three paragraphs, can be used provided proper acknowledgement is given.

The Rimini Centre for Economic Analysis (RCEA) was established in March 2007. RCEA is a private, nonprofit organization dedicated to independent research in Applied and Theoretical Economics and related fields. RCEA organizes seminars and workshops, sponsors a general interest journal The Review of Economic Analysis, and organizes a biennial conference: The Rimini Conference in Economics and Finance (RCEF) . The RCEA has a Canadian branch: The Rimini Centre for Economic Analysis in Canada (RCEACanada). Scientific work contributed by the RCEA Scholars is published in the RCEA Working Papers and Professional Report series.

The views expressed in this paper are those of the authors. No responsibility for them should be attributed to the Rimini Centre for Economic Analysis. 


\title{
A General Equilibrium Evaluation of the Employment Service
}

\author{
Miana Plesca* \\ Department of Economics \\ University of Guelph \\ miplesca@uoguelph.ca
}

This version: August 29, 2010

\begin{abstract}
This paper provides a general equilibrium evaluation of the Employment Service, also known as the Public Labor Exchange (PLX), a national program which facilitates meetings between job seekers and vacancies. The paper departs from the partial equilibrium framework of previous evaluations by constructing a dynamic general equilibrium matching model with the PLX as one search channel, and the other search channel comprising all other search methods. The PLX is a directed search channel in the sense that searchers are matched by skill levels. The model is calibrated to the U.S. PLX and to the U.S. labor market and is used to compute general and partial equilibrium impacts of the PLX. The findings are that (i) the partial equilibrium impacts are consistent with the empirical literature, but different from the general equilibrium ones; (ii) the standard assumption in the evaluation literature, that outcomes for agents who do not participate in a program are not directly affected by the program, does not hold for the PLX; (iii) the heterogeneity across and within worker skill levels plays an important role when computing aggregate impacts; and, (iv) equilibrium adjustments are driven by employers who post more high-skill vacancies when both search channels operate.
\end{abstract}

JEL Classification: J63, J64, J68, E24.

Keywords: Search Models, Program Evaluation, Public Employment Service, PLX,

General Equilibrium Impacts, Partial Equilibrium Impacts.

*I am grateful for excellent comments from the editor, two anonymous referees, Audra Bowlus, Berthold Herrendorf, Gueorgui Kambourov, Lance Lochner, Richard Rogerson, and Jeff Smith, as well as seminar participants at the 2003 Canadian Econometrics Study Group meeting, the 2005 Society for Economic Dynamics meeting, Arizona State, Bank of Canada, Guelph, HEC Montreal, McMaster, Ottawa, UQAM, Victoria, Waterloo, Western Ontario, Wilfrid Laurier, and York. This work was made possible by the facilities of the Shared Hierarchical Academic Research Computing Network (www.sharcnet.ca). 


\section{Introduction}

Government employment policies have shifted focus from a passive approach, such as unemployment insurance compensation, toward Active Labor Market Policy (ALMP) programs aimed at improving the skills and job market readiness of their participants. Examples of ALMP programs include classroom training in occupational skills, job search assistance (JSA), subsidized employment, or on-the-job training programs. Subsequently, a lot of work has been devoted to measuring the impact of employment and training programs. We have learned a lot about the substantive implications of the ALMP programs, as well as about the methodological challenges involved with obtaining program impacts.

The evaluation literature estimates the impact of a program by comparing the outcomes of program participants with the counterfactual outcomes that would have prevailed, had they not participated. For the most part, this literature has focused on estimating the partial equilibrium impacts of a program under the assumption that outcomes for non-participants are not affected by the operation of the program. This assumption is referred to as Stable Unit Treatment Value Assumption, or "SUTVA." While SUTVA may be reasonable for small-scale programs, where indirect effects are likely to be minimal, the partial equilibrium paradigm is no longer appropriate for large programs which have the potential to affect the outcomes of other agents in the economy besides participants. For example, a program successful at finding jobs for participants may do so at the expense of non-participants who would have obtained those jobs had the program not displaced them. Firms may also react to such an employment program by changing the number and skill mix of the vacancies they post.

The focus of this paper is on the Employment Service (ES), also known as the Public Employment Service (PES), or as the Public Labor Exchange (PLX). The PLX is a large-scale government-provided program which matches job searchers with vacancies. Each country has its own version of the Public Employment Service/PLX program, to which respective governments devote a significant amount of resources. ${ }^{1}$ The United States spends $0.17 \%$ of GDP on the PLX and Canada spends $0.21 \%$ of GDP. The fraction of GDP spent on ALMP programs is

\footnotetext{
${ }^{1}$ Throughout the paper, the Employment Service is referred to as the PLX, which is the preferred terminology in the U.S., to which the model is calibrated. Other studies, especially European ones, may address the PLX as ES or PES, but ultimately all terms refer to a similar program available in all OECD countries.
} 
higher for European countries in general. On the PLXs in particular, Sweden spends 0.25\% of GDP, the Netherlands spend $0.36 \%$ of GDP, Germany spends $0.22 \%$ of GDP, and the U.K. spends $0.18 \% .^{2}$ Further evidence of the significant scale of the PLX is seen from the very large number of PLX users. Over the period 1997-1999, the average number of workers registered on the PLX in the U.S. was 17.3 million each year; out of those, 11.2 million received "some reportable service" and 7.1 million were "referred to employment." In 1999, U.S. employers posted 7.5 million vacancies on the PLX. ${ }^{3}$

This paper is the first to provide a general equilibrium evaluation of the PLX. The contribution of this general equilibrium evaluation is threefold. First, the paper shows that general equilibrium adjustments are quantitatively important for the PLX as a large-scale program. The partial equilibrium impacts of the PLX computed from the model conform in magnitude with what has been documented elsewhere in the literature, and differ from the general equilibrium impacts; moreover, contrary to the standard assumption in partial equilibrium evaluation literature, the outcomes for agents who do not use the PLX actually do change because of the PLX. Second, since relatively few evaluations have investigated the role and impact of the PLX, the paper adds substantively to our understanding of the PLX as a labor market institution. Third, the model itself, while an extension of existing matching models set within the Mortensen-Pissarides framework, such as Albrecht and Vroman (2002), provides new insights into labor market adjustments from frictions on two search channels with different matching technologies. One search channel, the PLX, provides directed search to the extent that it help sort workers and vacancies by skill level. The other search channel is a random matching channel, comprising all other search methods, but more efficient at overall matching compared to the PLX. In this sense, the results here provide a quantitative example of the impact of directed search on wage inequality and welfare. ${ }^{4}$

The environment consists of unemployed workers who search for jobs and firms who post vacancies on two separate channels: the PLX channel, and an "everything else" channel, or

\footnotetext{
${ }^{2}$ Source: Table 2 in Boone and van Ours (2004). Averages for 1985-1999. Also see OECD (2001) for descriptions of the PLXs of most OECD countries; for instance Table 2 on page 51 lists how much each OECD country spends on their PLX as percent of total GDP.

${ }^{3}$ U.S. Department Of Labor, Employment and Training Administration Annual Report 1999 ETA9002-C.

${ }^{4}$ Shi (2002) is a theoretical reference for the impact of directed search on inequality; unlike Shi's model where search is directed by the firms' choice of the posted wage, here the directed search is embedded in the search technology on the PLX channel.
} 
the "Informal" channel. In every period, workers and firms choose whether to search on the Informal channel or on the PLX. Worker and firm heterogeneity is represented by two skill levels, high and low. Matches between workers and firms are productive when the worker skill endowments fall within the firm skill requirements. In order to model the operation of the PLX in the search market, PLX staff are assumed to separate workers and vacancies by skill level, subject to some error.

This provides an element of directed search in the sense that the PLX is providing a screening function that identifies, up to an error margin, the worker and vacancy types. Workers and vacancies then meet at random, but only within pools of similar types. The model is calibrated with agents searching on both channels, such that statistics from the model match those for the U.S. labor market and for the PLX along several dimensions, which include market tightness, the skill mix of searchers and vacancies, and unemployment duration. ${ }^{5}$

The general equilibrium impacts of the PLX are computed from the following experiment: the PLX is shut down and all agents are compelled to search on the Informal channel, the only one still available in the experimental economy. The impact of the PLX is computed as the difference in outcomes between the model economy, the one with both search channels available, and the simulated one, with the PLX shut down. This is the appropriate thought experiment since we are interested in the counterfactual outcome for PLX users in the absence of the PLX. Potential general equilibrium effects of the PLX stem from agents responding to the existence of the PLX search channel. By allowing employers to react to changes in the labor market due to the PLX, the number and skill mix of jobs and vacancies, and their duration, will change. Likewise, the existence of the PLX as a search option will change the speed and type of vacancies with which workers match. This affects in turn not only equilibrium unemployment and wages, but also the quality of matches, the welfare of workers and firms, and the labor productivity in the economy, in ways that would not be apparent in a partial equilibrium setting.

In particular, the general equilibrium impacts indicate that, in the absence of the PLX, high-skill workers would suffer a wage drop of $6.4 \%$ (this implies a positive wage impact of the PLX, computed as the difference in the wage with the PLX minus the wage without the

\footnotetext{
${ }^{5}$ Data for the calibration come from general labor surveys in the U.S., the Panel Study of Income Dynamics (PSID), the Current Population Survey (CPS), and the Job Openings and Labor Turnover Survey (JOLTS), as well as from public performance indicators for the PLX. Details on the calibration are provided in Section 5.
} 
PLX), while low-skill workers would gain $1.5 \%$ in wages (a negative wage impact of the PLX). Unemployment durations would decrease by six weeks for high-skill workers and by 2.7 weeks for low-skill workers in the absence of the PLX (this implies a positive duration impact of the PLX, only that in the case of unemployment duration, a negative impact would be preferred).

The partial equilibrium impacts computed from the model show the PLX generating a positive wage impact of about $1.6 \%$ on average and a small increase in the average duration of unemployment of 1.66 weeks. For non-participants, whose impacts are assumed to be zero in the standard partial equilibrium evaluation literature, the general equilibrium impacts indicate that the PLX has a positive impact on the mean wage (an increase of $1.1 \%$ ), together with an impact of 1.6 weeks increase in the mean unemployment duration. Thus, partial equilibrium impacts differ from the general equilibrium ones in the case of large national programs. This invites some caution when public policy is based on partial equilibrium estimates alone especially when considering heterogeneous impacts for various groups, high-skill and low-skill workers in this case.

The rest of the paper is organized as follows: Section 2 gives the institutional framework of the PLX and discusses existing literature on its evaluation. Section 3 introduces the model and solves for its equilibrium. Section 4 provides a link between the counterfactuals generated from the experiments conducted in the model and the corresponding parameters of interest in the program evaluation literature. Section 5 discusses the calibration procedure, and Section 6 presents the quantitative results for the general and partial equilibrium impacts. Section 7 concludes and offers some possible extensions.

\section{The Public Labor Exchange (PLX)}

\subsection{Institutional Framework}

PLX services are universally available with little enforced participation for either workers or firms. Job seekers have free access to listings of vacancies posted by employers and employer contact technology (such as computers or fax machines), and may receive technical guidance for preparing resumes. Unemployed individuals receiving unemployment insurance benefits have 
to register with the public agency, but their activity on the PLX is laxly monitored, if at all. ${ }^{6}$ For employers, the PLX provides vacancy registration and referrals of potential employees at little or no cost. Private firms are not mandated to list their vacancies on the PLX.

In order to match job seekers with employers, PLX computers run programs using client and vacancy characteristics to deliver a set of job openings for a job seeker or to provide a pool of qualified applicants for a vacancy. A referral entails a successful match by the PLX between job seekers and open vacancies. Referrals are based on information provided by job seekers "as is" without prior background checks by the PLX office. The relevant treatment considered here is receiving a job referral from the PLX; to discount passive users, simply registering with the PLX is not enough to be counted as treatment, unless a job referral results from of it.

\subsection{Partial Equilibrium Evaluations of the PLX}

\subsubsection{Reduced-form Evaluations of the PLX}

In the standard evaluation literature the impact of a program is measured in a reduced-form framework, by comparing actual outcomes with the estimated counterfactual of what would have happened to participants had they not participated, under the assumption that the program does not disrupt or influence the outcomes of non-participants. While ALMP programs inn general have been subjected to numerous evaluations, both experimental and nonexperimental, we know very little about the impact of the PLX on the wages and employment of its participants, despite the size and reach of public employment programs. Within this literature, only three studies have attempted to measure the impact of the PLX on the employment and wages of its users: Johnson, Dickinson, and West (1985), Katz (1991), and Jacobson and Petta (2000). This lack of knowledge comes in stark contrast with other ALMP programs, including Job Search Assistance (JSA) programs, which usually are also administered by the Employment Service. ${ }^{7}$

The three papers mentioned here focus on the U.S. PLX, and are set up in a standard

\footnotetext{
${ }^{6}$ See for instance Anderson (2001) for evidence on the variation of search requirements and monitoring of the unemployed across the U.S. Employment Agencies, who are responsible for the administration of the PLXs at the state level.

${ }^{7}$ See for instance the survey in O'Leary (2004) for more on the impact of JSA programs. One explanations why PLX programs have not been sufficiently evaluated is the fact that the PLX has universal access, which precludes any sort of experimental evaluation. Another strand of related literature, discussed in the next section, examines the impact of various job search methods, of which PLX could be one.
} 
reduced-form partial equilibrium framework. The documented impact of the PLX in reducing unemployment duration ranges from respectively 0.64 and 2.79 weeks of unemployment duration reduction for men and women (Johnson, Dickinson, and West (1985) using 1980 data) to 1.1 and 2.1 weeks of unemployment duration reduction in the states of Washington and Oregon (Jacobson and Petta (2000) using late-80s to mid-90s data). Similar modest impacts are documented for the wage outcomes, with men and women experiencing impacts of $\$-98$ and $\$ 325$ respectively over the six-month period following PLX intervention (Johnson, Dickinson, and West (1985)). These papers have neither generated a consensus regarding the role of the PLX in the labor market nor identified channels or mechanisms through which the PLX is helping (or not) its users.

\subsubsection{Partial Equilibrium Search Models of the PLX}

The other existing literature related to the PLX comes from partial equilibrium search models that investigate the effectiveness of various job-search strategies, including the PLX. Implicit in most reduced-form partial equilibrium studies is a model that takes the distribution of job openings as given and has job seekers choosing an optimal mix from a set of available job search methods: the PLX, networks of friends, direct employer contacts, newspaper ads, or private placement agencies. ${ }^{8}$ van Ours (1993) and Lindeboom, van Ours, and Renes (1994) argue that, compared to other search strategies, the Dutch PLX appears efficient for unemployed searchers. This is because employed searchers, who appear to search more effectively, use the PLX less than other channels, leaving unemployed searchers to face less competition on this channel. A similar argument is made by Fougere, Pradel, and Roger (2006) within the framework of

\footnotetext{
${ }^{8}$ One documented characteristic of PLX clients compared to workers who use other search methods is that PLX searchers are often low skilled and face serious barriers to re-employment (Bishop (1993) for the U.S, van Ours (1993) for the Netherlands, Gregg and Wadsworth (1996) for England, and Adison and Portugal (2002) for Portugal.); they may also receive lower wages upon finding employment (Cohen and Stevens (1989) for the U.S. and Osberg (1993) for Canada). This is consistent with PLX data which shows $89 \%$ of PLX users do not have post-secondary education, while in the population $61 \%$ of workers (and $75 \%$ of unemployed searching for a job) do not have post-secondary education. On the firm side, the literature examines the impact of different recruiting strategies on the time elapsed until vacancies are filled. From this literature it is not immediately apparent whether workers hired using other channels are more or less productive than workers hired through the PLX. Employers become more selective in the hiring procedures as the required levels of age, education, starting wages, and experience rise (Burdett and Cunningham (1998)). The PLX can help by providing a pre-screening service, facilitating contacts between workers and jobs with similar skills. This is consistent with van Ours and Ridder (1993) who argue that vacancy durations consist mostly of interviewing time, or Roper (1988) who finds the British PLX to be a faster recruiting channel than newspaper ads.
} 
a partial equilibrium search model with exogenous arrival rates of vacancies and endogenous search intensity. Despite the PLX lowering the search intensity, an exogenous increase in the arrival rate of vacancies would translate into an increase in exits from unemployment, proportionately larger for the low skill. Also related to the PLX, its crowding-out effect has been documented by Davidson and Woodbury (2000) in a chapter in the Jacobson and Petta (2000) study. The role of the PLX in the labor market is also modeled as changing the search intensity, thus reducing the job search cost for workers. With search cost parameters calibrated to wage and unemployment duration targets coming from the partial equilibrium estimates in Jacobson and Petta (2000) they find some reduction, although not too large, in the outcomes of non-users due to displacement. The role of the PLX in changing the search intensity, thus reducing the job search cost for workers, has also been modeled by Davidson and Woodbury (2000) in a chapter in the Jacobson and Petta (2000) study. Furthermore, Davidson and Woodbury (2000) document some reduction in the outcomes of non-users due to displacement by PLX users. ${ }^{9}$

While partial equilibrium search models bring important insights into the operation of the PLX and provide a useful benchmark for the partial equilibrium impacts of the PLX, they can miss important general equilibrium adjustments, such as the interaction between workers' search strategies and the vacancy-posting response of employers. ${ }^{10}$ In the case of the PLX, the partial equilibrium assumption is very likely to be violated, given the large scale of the program - and indeed the quantitative results from the model reveal that the general and partial equilibrium impacts are very different.

\footnotetext{
${ }^{9}$ Blundel, Dias, Meghir, and van Reenen (2004) also investigate the displacement effect (which they call "substitution") of the U.K. New Deal program, and cannot reject the null hypothesis of displacement effects. Their identification strategy is based on regional variation and differences between individuals eligible and ineligible for the program, assuming that the macro characteristics across regions (such as unemployment) are neither influenced by the program nor by differential external shocks.

${ }^{10}$ The very few papers which have approached program evaluation in a general equilibrium framework include Davidson and Woodbury (1993) and Lise, Seitz, and Smith (2004) who look at the displacement implications of programs offering bonus incentives for quitting unemployment or welfare; Heckman, Lochner, and Taber (1998) who account for the general equilibrium price adjustments of a universal tuition policy; and Albrecht, van den Berg, and Vroman (2005) who account for the skill acquisition of participants in a Swedish training program. Another trend in structural program evaluation, e.g. Wolpin and Todd (2006), uses experimental data to validate the behavioral implications of a structural model.
} 


\section{The Model}

Set within the Mortensen-Pissarides matching framework, the model departs from standard matching models by allowing two separate search channels on which workers and firms can interact: the PLX and the "Informal" channel that combines all other search channels. ${ }^{11}$ There is heterogeneity in worker and firm types, with an equilibrium distribution of searcher and vacancy types on the two channels. The general and partial equilibrium impacts of the PLX are quantified for outcomes which include employment, vacancies, wages, output, and a welfare measure embedded in value functions.

The model is set up in discrete time. Workers and firms are infinitely lived. Each period, workers and firms choose a search channel. Only unemployed workers search for jobs. Search costs are different for workers and firms across channels. The matching technology on the two channels is different in the sense that the PLX provides a pre-screening service for potential employers.

Heterogeneity is introduced by postulating two skill types: high and low. The distribution of worker skills is given exogenously. Each worker knows his/her own skill. Based on skill and labor market conditions, unemployed workers choose each period the search channel. The distribution of vacancy skill requirements is determined endogenously. Ex-ante identical firms decide whether to open a vacancy or not. The model only allows for one vacancy per firm. If the firm decides to open a vacancy, it also chooses the job skill requirement for the vacancy and on which channel to post it.

As in Albrecht and Vroman (2002), the productivity of a match is asymmetric: high-skill jobs can be filled only by high-skill workers, while low-skill jobs can be filled by either high-skill or low-skill workers. The productivity of a vacancy is determined by its skill requirement. A low-skill vacancy has the same productivity once filled, regardless of whether it is operated by a high-skill or a low-skill worker. High-skill positions are more productive, but also more difficult to fill, since low-skill workers are of no use to high-skill firms. ${ }^{12}$

\footnotetext{
${ }^{11}$ Other search channels include direct employer contacts, agents' personal connections, newspaper ads, etc.

${ }^{12}$ Albrecht and Vroman (2002) call the equilibrium with high-skill workers working in both type of jobs "crossskill equilibrium" and the equilibrium with high-skill workers working only in high-skill jobs "segmented-skill equilibrium." Following empirical evidence from workers' education and wages, my focus here is on the crossskill equilibrium. Also note that, while I focus on an equilibrium where there is a mass of workers and vacancies on each channel, I cannot rule out the possibility of multiple equilibria, where workers and/or vacancies could
} 
Let subscript $j$ denote the search channel, with $j=\{1,2\}$, where the Informal channel is subscripted by 1 and the PLX channel is subscripted by 2. Workers are of skill type $x$, high $(x=H)$ or low $(x=L)$. Vacancies have a skill requirement $y$, high $(y=H)$ or low $(y=L)$. Each period, unemployed workers decide on which channel to search for a job and firms decide on which channel to post a vacancy. Productive matches continue until exogenously destroyed. There are two different exogenous job destruction rates for high-skill and for low-skill jobs, $\delta^{H}$ and $\delta^{L}$. Agents discount the future at rate $\beta$. Wages are determined by workers and firms splitting the surplus of a productive match in a symmetric Nash bargaining framework.

\subsection{The Worker's Problem}

Unemployed workers receive per period benefits $b$. These benefits can be seen as an aggregate measure of home production, value of extra leisure, and unemployment compensation. The cost of search $c_{j}$ is the same for high-skill and low-skill unemployed workers, but may differ across search channels.

The unemployment value for a type $x$ worker searching on channel $j$, is given by:

$$
\begin{aligned}
U_{j t}(x) & =b-c_{j} \\
& +\beta\left\{\sum_{y=\{H, L\}} p_{j t}(x, y) \max \left[U_{t+1}(x), W_{j t+1}(x, y)\right]+\left[1-\sum_{y=\{H, L\}} p_{j t}(x, y)\right] U_{t+1}(x)\right\} \\
& \quad \text { for } j=\{1,2\}, \quad x=\{H, L\}
\end{aligned}
$$

where $p_{j t}(x, y)$ is the per-period probability that an unemployed worker of skill $x$ meets a vacancy of skill requirement $y$ on channel $j$. $U_{t+1}(x)$ is the period $t+1$ value of unemployment for a type $x$ worker. It is independent of the channel choice $j$ because workers have the option of choosing the search channel at the beginning of every period. Therefore, $U_{t+1}(x)$ is given by:

$$
U_{t+1}(x)=\max _{j=\{1,2\}}\left\{U_{j t+1}(x)\right\} \quad \text { for } x=\{H, L\}
$$

Given the choice of a search channel each period, it must be the case that the value of unemployment is the same on both channels, $U_{1 t}(x)=U_{2 t}(x)=U_{t}(x)$.

segregate on one or the other search channel. More details on the corner solutions are provided in Section 3.6 and also in the Appendices. 
When employed, workers receive per period wage compensation $w_{j t}(x, y)$ and face the exogenous probability $\delta^{y}$ of losing their job and becoming unemployed. The value of a job match for a worker of type $x$ whose search on channel $j$ has succeeded in finding a job of skill $y$ is:

$$
\begin{array}{r}
W_{j t}(x, y)=w_{j t}(x, y)+\beta\left[\left(1-\delta^{y}\right) W_{j t+1}(x, y)+\delta^{y} U_{t+1}(x)\right], \\
\text { for } j=\{1,2\}, \quad x=\{H, L\}, \quad y=\{H, L\} .
\end{array}
$$

\subsection{The Firm's Problem}

A firm can post either high-skill $y=H$ or low-skill $y=L$ vacancies. The cost of posting a vacancy of skill requirement $y$ on channel $j$ is $k_{j}(y)$. The value of opening a vacancy of skill requirement $y$ on channel $j$ is given by:

$$
\begin{aligned}
V_{j t}(y)= & -k_{j}(y) \\
+ & \beta\left\{\sum_{x=\{H, L\}} q_{j t}(x, y) \max \left[V_{t+1}(y), J_{j t+1}(x, y)\right]+\left[1-\sum_{x=\{H, L\}} q_{j t}(x, y)\right] V_{t+1}(y)\right\} \\
& \text { for } j=\{1,2\}, y=\{H, L\}
\end{aligned}
$$

where $q_{j t}(x, y)$ is the probability that a vacancy of skill requirement $y$ meets an unemployed worker of type $x$ searching on channel $j$ in period $t . J_{j t+1}(x, y)$ is the firm's value from a productive match between a skill $y$ vacancy posted on channel $j$ meeting a type $x$ worker in period $t$. Each period, firms can choose on which channel to post their vacancy:

$$
V_{t+1}(y)=\max _{j=\{1,2\}}\left\{V_{j t+1}(y)\right\} .
$$

Like in the worker case, the choice of a posting channel implies that the value of vacancy must be the same on either channel, and therefore the subscript $j$ may be omitted from the value function. When first opening a vacancy, the firm decides its type. Assign to the initial skill choice the value $V_{0}=\max _{y=\{H, L\}}\left\{V_{0}(y)\right\}$.

The technology is such that the output of a productive match is the job skill requirement, as long as the worker skill is at least as high as the job skill requirement, otherwise no output is produced:

$$
\begin{aligned}
f(x, y)=f(y) & \text { if } x \geq y \\
0 & \text { if } x<y .
\end{aligned}
$$


If productive, a filled vacancy generates for the firm:

$$
\begin{gathered}
J_{j t}(x, y)=f(y)-w_{j t}(x, y)+\beta\left\{\left(1-\delta^{y}\right) J_{j t+1}(x, y)+\delta^{y} V_{0}\right\} \\
\text { for } j=\{1,2\}, \quad x=\{H, L\}, \quad y=\{H, L\}
\end{gathered}
$$

where $\delta^{y}$ is the probability of an exogenous job destruction for a job of productivity $y$.

\subsection{Wage Determination}

Every period, on each channel $j=\{1,2\}$, the net surplus from a job match between a type $x$ worker and a skill $y$ vacancy, $S_{j}(x, y)=\left[W_{j t}(x, y)-U_{t}(x)\right]+\left[J_{j t}(x, y)-V_{t}(y)\right]$, is split by a Nash bargaining rule, giving wages $w_{j t}(x, y)$ as the solution to:

$$
\max \left[W_{j t}(x, y)-U_{t}(x)\right]^{s}\left[J_{j t}(x, y)-V_{t}(y)\right]^{1-s}, \quad \text { for } x=\{H, L\}, \quad y=\{H, L\}
$$

where $s$ is the worker's bargaining power. The maximization implies:

$$
(1-s)\left[W_{j t}(x, y)-U_{t}(x)\right]=s\left[J_{j t}(x, y)-V_{t}(y)\right], \quad \text { for } x=\{H, L\}, \quad y=\{H, L\}
$$

\subsection{Steady-state Flow Conditions}

To simplify notation, I anticipate here an equilibrium feature of the model, stationarity. Since only steady-state equilibrium quantities are of interest, the $t$ subscripts can be suppressed from all definitions, including value functions and equilibrium unemployment, employment and vacancies.

Let $u_{H}$ denote the number of high-skill unemployed and $u_{L}$ the number of low-skill unemployed. Let $u_{j H}$ and $u_{j L}$ be the number of high-skill and low-skill unemployed on channel $j$, where $j=\{1,2\}$. The number of high-skill and low-skill employed from channel $j$ is respectively $e_{j H}=e_{j H H}+e_{j H L}$ and $e_{j L}=e_{j L L}$, where being employed from channel $j$ means the worker and firm were searching on channel $j$ when they met and agreed to enter the productive relationship. ${ }^{13}$

\footnotetext{
${ }^{13}$ Although they represent stocks, the employment measures are denoted by small letters to avoid confusion with value functions, which are denoted by capital letters. The total number of unemployed on channel $j$ is $u_{j H}+u_{j L}$. The total number employed from the Informal channel is $e_{1 H H}+e_{1 H L}+e_{1 L L}$, while from the PLX it is $e_{2 H H}+e_{2 H L}+e_{2 L L}$. A high-skill worker in a high-skill job $e_{H L}$ faces a lower job destruction probability $\delta^{H}$ compared to a high-skill worker in a low-skill job $e_{H L}$ facing a job destruction probability of $\delta^{L}$. The high-skill labor force is $e_{H}+u_{H}$, and the low-skill labor force is $e_{L}+u_{L}$.
} 
For each worker type $x=\{H, L\}$ on each channel $j=\{1,2\}$ the number of employed individuals of skill $x$ who become unemployed after working in a job $y$ is equal to the number of unemployed individuals of skill $x$ who find a job $y$, resulting in the following flow conditions:

$$
e_{j(x, y)}=u_{j x} \frac{p_{j}(x, y)}{\delta^{y}} \quad \text { for } j=\{1,2\}, \quad x=\{H, L\}, y \leq x
$$

where $p_{j}(x, y)$ is the probability that a worker of type $x$ meets a vacancy of skill requirement $y$ while searching on channel $j$. Since there are no productivity dynamics, there are no incentives for workers or vacancies to switch between search channels in equilibrium and, therefore, there are no equilibrium flows of either workers or vacancies between the two channels.

There are two more equations, one equation which normalizes the total labor force to one:

$$
\sum_{j=\{1,2\}, x=\{H, L\}} e_{j x}+u_{j x}=1
$$

and another equation derived from the exogenous distribution of skill types $\eta:{ }^{14}$

$$
\sum_{j=\{1,2\}} e_{j H}+u_{j H}=\eta .
$$

\subsection{Matching Technology}

Let $v_{j H}$ and $v_{j L}$ denote high-skill and low-skill vacancies for each channel $j=1,2$. Define the following quantities:

Market tightness: ${ }^{15}$

$$
\theta_{j}=\frac{v_{j}}{u_{j}}=\frac{v_{j H}+v_{j L}}{u_{j H}+u_{j L}} .
$$

Fraction of low-skill searchers (unemployed):

$$
\gamma_{j}=\frac{u_{j L}}{u_{j H}+u_{j L}}
$$

Fraction of low-skill vacancies:

$$
\phi_{j}=\frac{v_{j L}}{v_{j H}+v_{j L}} .
$$

\footnotetext{
${ }^{14}$ Only the overall fraction of high-skilled labor force is exogenous. The fraction of high-skilled and low-skilled unemployed searching on each channel is endogenous.

${ }^{15}$ Market tightness can be defined separately for high-skill and for low-skill, or jointly for both skills. The joint definition provided here is some weighted average of the separate tightness for high- and low-skill. Separate tightness measures by skill level will be used when describing matching on the PLX channel.
} 
The existence of a matching function is prompted by the spatial and/or temporal frictions in the labor market that hamper meetings between workers and employers. Denote by $M_{j}\left(u_{j}, v_{j}\right)$ the number of random meetings generated in a market where $u_{j}$ workers and $v_{j}$ vacancies are searching for a match.

The meeting technology is homogeneous of degree one in $u$ and $v$, increasing and concave in both arguments. ${ }^{16}$ The most commonly used functional form for the meeting technology $M(u, v)$ is Cobb-Douglas, because it performs well when taken to the data - see for instance the survey in Petrongolo and Pissarides (2001). To ensure that the meeting probabilities are in the $(0,1)$ interval, I follow den Haan, Ramey, and Watson (2000) and use a slightly different functional form, also homogeneous of the first degree: ${ }^{17}$

$$
\begin{aligned}
M_{j}\left(\theta_{j}\right) & =\lambda_{j} \frac{u_{j} v_{j}}{\left(u_{j}^{\alpha}+v_{j}^{\alpha}\right)^{\frac{1}{\alpha}}} \\
& =\lambda_{j} \frac{\theta_{j}}{\left(1+\theta_{j}^{\alpha}\right)^{\frac{1}{\alpha}}} \cdot u_{j}=\lambda_{j} \frac{1}{\left(1+\theta_{j}^{\alpha}\right)^{\frac{1}{\alpha}}} \cdot v_{j} \\
& =m\left(\theta_{j}\right) \cdot u_{j}
\end{aligned}
$$

where $\theta_{j}=\frac{v_{j}}{u_{j}}$ is the overall market tightness on channel $j=\{1,2\}, \alpha \in(0,1)$ is a matching parameter, and $m\left(\theta_{j}\right)=\lambda_{j} \frac{\theta_{j}}{\left(1+\theta_{j}^{\alpha}\right)^{\frac{1}{\alpha}}}$.

The matching efficiency parameter $\lambda$ is going to be important in the calibrated version of the model. Because the PLX provides a sorting service by skill level, it is obviously an attractive mechanism. The calibrated equilibrium will have a measure of individuals and vacancies of each skill on both channels. To achieve a distribution that is consistent with observable data, some matching advantage must be allowed for the Informal channel, otherwise all workers and vacancies might migrate on the PLX channel. ${ }^{18}$ Since it is only the relative matching efficiency that matters, the matching efficiency for channel 2 is set to one, $\lambda_{2} \equiv 1$, while the value for

\footnotetext{
${ }^{16}$ The decision of a worker to search for a job generates two opposing externalities. On the one hand, the more workers searching for a job, the easier for a firm to fill a vacancy. On the other hand, the more workers searching, the more difficult it is for a worker to meet with a vacancy. If the effect of these two externalities cancels out, then it can be argued that the matching technology only depends on the ratio of searching workers to posted vacancies, therefore justifying the widely-held assumption that the matching function exhibits constant returns to scale.

${ }^{17}$ In a continuous time setting this would not be an issue, since the rates of contact computed in the continuous case do not have to lie between 0 and 1.

${ }^{18} \mathrm{An}$ equilibrium with matching efficiencies set to 1 on both channels could not be calibrated to match statistics in the data, for reasonable values of the probabilities that the PLX misidentifies worker and vacancy skills.
} 
the matching efficiency $\lambda_{1}$ is determined in calibration. While in principle the calibration could pick a parameter $\lambda_{1}<1$, for the reason outlined above we should expect a value of $\lambda_{1}>1$.

\subsubsection{Meeting on the Informal Channel}

The probability that a worker of skill level $x$ meets a vacancy of skill requirement $y$ does not depend on the worker skill $x$, since the matching is random and all workers have the same chance of meeting a vacancy. High-skill and low-skill workers are equally likely to meet a high-skill vacancy $p_{1}(H, H)=p_{1}(L, H)$ and, likewise, they are equally likely to meet a low-skill vacancy $p_{1}(H, L)=p_{1}(L, L)$. The probability for a low-skill worker to meet a high-skill vacancy $p_{1}(L, H)$ is irrelevant, since such a productive match will never form.

Reciprocally, the probability that a vacancy of skill requirement $y$ meets a worker of skill level $x$ does not depend on the job skill requirement $y$. A high-skill vacancy and a low-skill vacancy are equally likely to meet a high-skill worker $q_{1}(H, H)=q_{1}(H, L)$, or equally likely to meet a low-skill worker $q_{1}(L, H)=q_{1}(L, L)$. As before, $q_{1}(L, H)$ is irrelevant since a low-skill worker $x=L$ does not generate any positive output when in a high-skill job $y=H$.

These meeting probabilities are respectively:

$$
\begin{aligned}
p_{1}(H, H) & =\frac{M_{1}(u, v)}{u_{1}} \cdot \frac{v_{1 H}}{v_{1 H}+v_{1 L}}=m\left(\theta_{1}\right)\left(1-\phi_{1}\right) \\
p_{1}(H, L)=p_{1}(L, L) & =\frac{M_{1}(u, v)}{u_{1}} \cdot \frac{v_{1 L}}{v_{1 H}+v_{1 L}}=m\left(\theta_{1}\right) \phi_{1} \\
q_{1}(H, H)=q_{1}(H, L) & =\frac{M_{1}(u, v)}{v_{1}} \cdot \frac{u_{1 H}}{u_{1 H}+u_{1 L}}=\frac{m\left(\theta_{1}\right)}{\theta_{1}}\left(1-\gamma_{1}\right) \\
q_{1}(L, L) & =\frac{M_{1}(u, v)}{v_{1}} \cdot \frac{u_{1 L}}{u_{1 H}+u_{1 L}}=\frac{m\left(\theta_{1}\right)}{\theta_{1}} \gamma_{1}
\end{aligned}
$$

\subsubsection{Meeting on the PLX Channel}

On the PLX channel, the meeting technology is assortative to the extent that PLX personnel refer high-skill workers to high-skill vacancies and low-skill workers to low-skill vacancies. While it is assumed that PLX personnel do the best they can to match job seekers and vacancies by their respective characteristics, there is room for error. ${ }^{19}$ Subject to the identification error, the

\footnotetext{
${ }^{19}$ Mistakes from PLX staff in identifying skills can be genuine, either accidental or because the workers attempt to misrepresent their qualifications. One could also imagine that PLX staff, attempting to manipulate referral and placement statistics, on which their own job performance may be assessed, could willfully "mistake" worker skills or job requirements.
} 
PLX separates workers and vacancies on the PLX channel into two markets, or pools, one for high-skill matches and one for low-skill matches. The effective number of workers and vacancies searching on the PLX channel is given not by their true type, but by how the PLX perceives the skill type.

Let $d_{w}$ be the probability that the PLX mistakenly identifies the type of a worker, and $d_{v}$ the probability that the PLX mistakenly identifies the type of a vacancy. Denoting by tilde the effective measures for workers and vacancies on the PLX, the following identities hold:

$$
\begin{aligned}
& \widetilde{u}_{2 H}=\left(1-d_{w}\right) u_{2 H}+d_{w} u_{2 L} \text { and } \widetilde{u}_{2 L}=d_{w} u_{2 H}+\left(1-d_{w}\right) u_{2 L} \\
& \widetilde{v}_{2 H}=\left(1-d_{v}\right) v_{2 H}+d_{v} v_{2 L} \text { and } \widetilde{v}_{2 L}=d_{v} v_{2 H}+\left(1-d_{v}\right) v_{2 L} .
\end{aligned}
$$

A high-skill worker on the PLX is referred to a high-skill vacancy either if the PLX correctly identifies both the worker and the vacancy as high-skill types, or if the PLX misidentifies both the worker and the vacancy as low-skill types. The probability that a worker of skill $x=H$ searching on the PLX channel is referred to a vacancy of skill $y=H$ is therefore the sum of two terms. The first term is the probability that the $x=H$ worker is correctly identified, times the number of matches per searcher in the $H$ pool, times the probability that the matched vacancy from the $H$ pool is also correctly identified as a $y=H$ vacancy. Likewise, the second term is the probability that the $x=H$ worker is misidentified as low-skill, times the number of matches per searcher in the $L$ pool, times the chance that the matched vacancy from the $L$ pool happens to be a misidentified $y=H$ vacancy:

$$
p_{2}(H, H)=\left(1-d_{w}\right) \cdot \frac{\widetilde{M}_{2 H}}{\widetilde{u}_{2 H}} \cdot \frac{\left(1-d_{v}\right) v_{2 H}}{\widetilde{v}_{2 H}}+d_{w} \cdot \frac{\widetilde{M}_{2 L}}{\widetilde{u}_{2 L}} \cdot \frac{d_{v} v_{2 H}}{\widetilde{v}_{2 L},}
$$

where $\widetilde{M}_{2 H}$ is the meeting function on the PLX pool identified as high-skill and $\widetilde{M}_{2 L}$ is the meeting function on the PLX pool identified as low-skill. Likewise, the probability that a worker of skill $x=H, L$ searching on the PLX channel is referred to a low-skill $y=L$ vacancy is:

$$
\begin{aligned}
& p_{2}(H, L)=\left(1-d_{w}\right) \cdot \frac{\widetilde{M}_{2 H}}{\widetilde{u}_{2 H}} \cdot \frac{d_{v} v_{2 L}}{\widetilde{v}_{2 H}}+d_{w} \cdot \frac{\widetilde{M}_{2 L}}{\widetilde{u}_{2 L}} \cdot \frac{\left(1-d_{v}\right) v_{2 L}}{\widetilde{v}_{2 L}} \\
& p_{2}(L, L)=d_{w} \cdot \frac{\widetilde{M}_{2 H}}{\widetilde{u}_{2 H}} \cdot \frac{d_{v} v_{2 L}}{\widetilde{v}_{2 H}}+\left(1-d_{w}\right) \cdot \frac{\widetilde{M}_{2 L}}{\widetilde{u}_{2 L}} \cdot \frac{\left(1-d_{v}\right) v_{2 L}}{\widetilde{v}_{2 L}}
\end{aligned}
$$

The same applies on the vacancy side: vacancies are matched with the same worker type if the vacancy and the worker are either both correctly identified or both misidentified. Vacancies are 
matched with the opposite worker type if either one is misidentified. This leads to the following meeting probabilities:

$$
\begin{aligned}
q_{2}(H, H) & =\left(1-d_{v}\right) \cdot \frac{\widetilde{M}_{2 H}}{\widetilde{v}_{2 H}} \cdot \frac{\left(1-d_{w}\right) u_{2 H}}{\widetilde{u}_{2 H}}+d_{v} \cdot \frac{\widetilde{M}_{2 L}}{\widetilde{v}_{2 L}} \cdot \frac{d_{w} u_{2 H}}{\widetilde{u}_{2 L}} \\
q_{2}(H, L) & =\left(1-d_{v}\right) \cdot \frac{\widetilde{M}_{2 H}}{\widetilde{v}_{2 H}} \cdot \frac{d_{w} u_{2 L}}{\widetilde{u}_{2 H}}+d_{v} \cdot \frac{\widetilde{M}_{2 L}}{\widetilde{v}_{2 L}} \cdot \frac{\left(1-d_{w}\right) u_{2 L}}{\widetilde{u}_{2 L}} \\
q_{2}(L, L) & =d_{v} \cdot \frac{\widetilde{M}_{2 H}}{\widetilde{v}_{2 H}} \cdot \frac{d_{w} u_{2 L}}{\widetilde{u}_{2 H}}+\left(1-d_{v}\right) \cdot \frac{\widetilde{M}_{2 L}}{\widetilde{v}_{2 L}} \cdot \frac{\left(1-d_{w}\right) u_{2 L}}{\widetilde{u}_{2 L}}
\end{aligned}
$$

Denote the effective market tightness by:

$$
\widetilde{\theta}_{2 x}=\frac{\widetilde{v}_{2 x}}{\widetilde{u}_{2 x}} \quad \text { for } x=\{H, L\}
$$

where $\widetilde{u}_{2 x}$ is the number of unemployed identified by the PLX as having skill $x=\{H, L\}$ and $\widetilde{v}_{2 y}$ is the number of vacancies identified by the PLX as having skill requirement y. Using these effective quantities, the meeting functions on the PLX channel, homogeneous of degree one and increasing in their arguments, have the same functional form as the meeting function on the Informal channel:

$$
\begin{aligned}
\widetilde{M}_{2 x}\left(\widetilde{\theta}_{2 x}\right) & =\frac{\widetilde{u}_{2 x} \cdot \widetilde{v}_{2 x}}{\left(\widetilde{u}_{2 x}^{\alpha}+\widetilde{v}_{2 x}\right)^{\frac{1}{\alpha}}} \\
& =\frac{\widetilde{\theta}_{2 x}}{\left(1+\widetilde{\theta}_{2 x}\right)^{\frac{1}{\alpha}}} \cdot \widetilde{u}_{2 x}=\frac{1}{\left(1+\widetilde{\theta}_{2 x}\right)^{\frac{1}{\alpha}}} \cdot \widetilde{v}_{2 x} \\
& =\widetilde{m}_{2 x} \cdot \widetilde{u}_{2 x},
\end{aligned}
$$

where $\widetilde{m}_{2 x}=\frac{\widetilde{\theta}_{2 x}}{\left(1+\widetilde{\theta}_{2 x}^{\alpha}\right)^{\frac{1}{\alpha}}}, \alpha \in(0,1)$ a matching parameter, and $d_{w}$ and $d_{v}$ respectively the fraction of searching workers and vacancies misidentified by the PLX. ${ }^{20}$

Appendix I presents formulas for meeting probabilities on the PLX channel, where the usual quantities - "true" measures of high and low-skilled unemployed workers and vacancies and market tightness on the PLX channel - are replaced with the "effective" measures described above, $\widetilde{u}_{2 x}, \widetilde{v}_{2 x}$ and $\widetilde{\theta}_{2 x}$.

\footnotetext{
${ }^{20}$ Note that the matching efficiency parameter $\lambda_{2}$ has been omitted from the formula, since it is being normalized to 1 in any case.
} 


\subsection{Equilibrium}

The recursive steady-state equilibrium is an array $\left\{U, W_{j}, V, J_{j}, w_{j}, p_{j}, q_{j}\right\}$ such that:

1. Given $w_{j}, p_{j}$, and $q_{j}$, the value functions solve the Bellman equations corresponding to workers' and firms' maximizing behavior.

2. Free entry implies that the value of all open vacancies is zero.

3. Steady-state flow conditions hold on each channel.

4. Given value functions $U, W_{j}, V$, and $J_{j}$, wages are determined as a Nash bargaining outcome.

5. Expectations about meeting probabilities are realized in equilibrium.

Upon a meeting between a type $x$ worker and a skill requirement $y$ vacancy, a productive match is formed if and only if the match can generate a positive surplus in present value discounted terms. In equilibrium, this implies that the value of the job match is positive, since free entry drives the value of a vacancy to zero: $J(x, y)>0$, or, equivalently, that the present value of working is higher than the present value of being unemployed: $W(x, y)>U(x)$.

Productive matches between low-skill workers and high-skill vacancies have been ruled out by the production function assumption. Low-skill workers always engage in productive matches with low-skill vacancies upon meeting such vacancies, and high-skill workers always engage in productive matches with high-skill vacancies. Furthermore, as mentioned earlier, the focus here is on the equilibrium where high-skill workers also engage in productive matches upon meeting low-skill vacancies. ${ }^{21}$

There are several possible ways for workers and vacancies to interact on the two search channels. Because it is the equilibrium observed in the real world, the model is calibrated to the interior solution where workers and vacancies of both types search on both channels;

\footnotetext{
${ }^{21}$ Albrecht and Vroman (2002) allow for two type of equilibria, "cross-skill" (the one considered here) and "segmented-skill", an equilibrium outcome where high-skill workers produce only in high-skill jobs. For them the two type of equilibria is essential as the purpose of their paper is to show that using a Mortensen-Pissarides matching framework together with a particular production technology can explain income inequality, both within $(w(H, H)>w(H, L))$ and between $(w(H, L)>w(L, L))$ skill groups. Here, segmented-skill matching is just a potential extension, whose equilibrium solution is available from the author upon request.
} 
I call this the full-mixing equilibrium. The solution is discussed in the following Section 3.7. Full details of the derivations, together with formulas for all other quantities of interest, are presented in Appendix II.

Three other possible equilibria are discussed in detail in Appendices III and IV: a separation case, when all workers and vacancies choose to migrate on the informal search channel; the reciprocal case, when all agents find it optimal to migrate on the PLX search channel; and, finally, a partial mixing case, when high-skill workers search only on the Informal channel and low-skill workers search only on the PLX. ${ }^{22}$

These other equilibria are interesting not only for theoretical purposes, but also for the quantitative implications of the analysis, since the different experiments performed on the fullmixing model may result in some of the equilibria described here.

\subsection{Solving for a Stationary Equilibrium}

The solution to the model comes from the following conditions: the surplus from a productive match is split by the Nash bargaining rule; employment is worthwhile for workers $W>U$ (accounting for the outside option of an employed worker); and employment is worthwhile for vacancies $J>V=0$ (accounting for the outside option of a filled vacancy). Finally, equilibrium stationarity flow conditions are used to compute other quantities of interest such as wages, employment, and unemployment for each skill type on each search channel.

The system of equations for solving each model can be reduced to three equations in three unknowns: market tightness $(\theta)$, fraction of low- skill unemployed $(\gamma)$, and fraction of low-skill vacancies $(\phi)$. In particular, in the baseline case of a mixing equilibrium (workers of both skills searching on both channels), the equilibrium conditions can be reduced to a system of equations in six unknowns: the market tightness on both search channels, $\theta_{1}$ and $\theta_{2}$; the fraction of low-skill vacancies, $\phi_{1}$ and $\phi_{2}$; and the fraction of unemployed low-skill searchers, $\gamma_{1}$ and $\gamma_{2}$.

\footnotetext{
${ }^{22}$ The possibility of multiple equilibria cannot be ruled out. Because of the complicated nonlinearities introduced by the matching technology on the PLX channel, a closed-form solution is impossible to obtain for any equilibrium. Furthermore, no analytical conditions can be derived that would allow to separate regions of the parameter space consistent with only one or another type of equilibrium. The behavioural implications of the separating and partial mixing equilibria are summarized not only by equilibrium outcomes, but also by the inequalities that need to be satisfied for these limiting cases to occur, as described in the Appendices.
} 


\subsubsection{Vacancies and Workers Choose to Engage in Production upon Meeting}

By substituting the free entry condition $V(y)=0$ in the value of a productive match for a firm, equation (4), we get:

$$
J_{j}(x, y)=\frac{f(y)-w_{j}(x, y)}{B^{y}}
$$

where for simplicity I denote $B^{y}=1-\beta+\beta \delta^{y}$. On the worker side, stationarity in the value of a productive match for the worker, equation (2), implies:

$$
W_{j}(x, y) B^{y}=U(x) \beta \delta^{y}+w_{j}(x, y) .
$$

Substituting for free entry $V(y)=0$ in the Nash bargaining rule (5) and replacing the expressions for $W_{j}(x, y)$ and $J_{j}(x, y)$ from above we obtain:

$$
(1-s)\left[\frac{U(x) \beta \delta^{y}+w_{j}(x, y)}{B^{y}}-U(x)\right]=s \frac{f(y)-w_{j}(x, y)}{B^{y}},
$$

or, after some manipulation,

$$
f(y)-w_{j}(x, y)=(1-s)[f(y)-(1-\beta) U(x)]
$$

Since the outside option of a worker in a productive match $U(x)$ does not depend on the channel where the match occurred, it follows that in equilibrium the value of unemployment, and therefore the wages paid to a type $x$ worker in a skill $y$ job, must be the same across each channel: $w_{j}(x, y) \equiv w(x, y)$.

There are three wages being paid in equilibrium: $w(H, H), w(H, L)$, and $w(L, L)$. High-skill workers in low-skill jobs receive a different wage than low-skill workers in the same job because they have a different outside option of being unemployed, $U(H) \neq U(L)$. High-skill workers in high-skill jobs receive a higher wage than high-skill workers in low-skill jobs because the productivity of high-skill jobs is higher $f(H)>f(L){ }^{23}$

From (19) and (21) we have that

$$
J(x, y)=\frac{f(y)-w(x, y)}{B^{y}}=\left(\frac{1-s}{B^{y}}\right)[f(y)-(1-\beta) U(x)] .
$$

\footnotetext{
${ }^{23}$ Note that, since $U(x)$ does not depend on the job skill requirement $y,(21)$ implies that $w(H, H)-w(H, L)=$ $s(f(H)-f(L)$. It shows that the wage premium for a high-skill worker in a high-skill job relative to the lowskill job is the worker's share of the productivity differences between the high- and low-skill jobs.
} 
Since workers and firms can choose what channel to use, the present discounted values of a match for the worker $W_{j}(x, y)$ or for the firm $J_{j}(x, y)$ have to be the same on each channel, $W_{j}(x, y) \equiv W(x, y)$ and $J_{j}(x, y) \equiv J(x, y)$. Therefore, in equilibrium all value functions, as well as wages, are the same across the two search channels.

\subsubsection{Outside Option: Employment Value for the Worker}

Given that productive matches are formed upon meeting, the value of a job is higher than the value of unemployment and thus $\max [U(x), W(x, y)]=W(x, y)$. Re-writing equation (1) by substituting $\max [U(x), W(x, y)]=W(x, y)$ and then replacing $W(x, y)-U(x)$ with the corresponding expressions from (20) and (21) we get:

$$
\begin{aligned}
& U(x)=\left(b-c_{j}\right)+\beta\left\{s \sum_{y \leq x} \frac{p_{j}(x, y)[f(y)-(1-\beta) U(y)]}{B^{y}}\right\}+\beta U(x) \\
& \text { for } j=\{\text { Informal, } P L X\}, \quad x=\{H, L\}
\end{aligned}
$$

If channel $j$ is chosen by type $x$ worker, the condition that workers form a productive match upon meeting a vacancy on channel jgives the following value of unemployment:

$$
\begin{array}{r}
(1-\beta) U(x)=\frac{\left(b-c_{j}\right)+\beta s \sum_{y \leq x} \frac{p_{j}(x, y) f(y)}{B^{y}}}{1+\beta s \sum_{y \leq x} \frac{p_{j}(x, y)}{B^{y}}} \\
\text { for } j=\{1,2\}, \quad x=\{H, L\}
\end{array}
$$

\subsubsection{Outside Option: Employment Value for the Firm}

Under the assumption that there are no corner solutions in terms of no vacancies being offered (i.e. the value of a vacancy is not negative) equation (4) can be re-written using the condition that the value of a vacancy is zero in a free entry equilibrium. Substitute $V(y)=0$ and expression $(22)$ for $J(x, y)$ in the value of a vacancy given by equation (4):

$$
\begin{array}{r}
0=-k_{j}(y)+\beta \sum_{x \geq y} q_{j}(x, y) \frac{(1-s)}{B^{y}}[f(y)-(1-\beta) U(x)] \\
\text { for } j=\{1,2\}, \quad y=\{H, L\}
\end{array}
$$

Equations (23) and (24) give the interior equilibrium solution. Appendix II presents details for deriving the implicit form solution after $U(H)$ and $U(L)$ have been eliminated between the above equations. Having solved for the equilibrium $\theta_{j}, \phi_{j}$ and $\gamma_{j}$, the stationarity flow 
conditions (6), (7) and (8) give the other equilibrium quantities of interest: wages, employment and unemployment, and the output produced $Y$. Their respective expressions are given in Appendix II.1.

\section{Parameters of Interest in the General Equilibrium Eval- uation of the PLX}

In the present formulation, "treatment" for the worker means receiving a job referral from PLX staff; for the firm, it means listing a vacancy on the PLX. Workers and firms self-select into treatment by choosing whether to use the PLX search channel or the Informal channel.

To compute the counterfactual outcome (what would have happened had the worker or the firm not received the PLX "treatment") the thought experiment is to have the worker, or the firm, search on the Informal channel instead. Because of the large size of the PLX, however, a general equilibrium counterfactual should take into account all adjustments that would happen if all searchers were to move on the Informal channel instead. In other words, the correct general equilibrium counterfactual needed to determine the role of the PLX in the labor market would be to have the PLX shut down and see how equilibrium quantities would change in a world where the PLX did not operate. By contrast, the thought experiment for the partial equilibrium impact takes a marginal agent from the PLX and determines what would have happened to this one agent had s/he gone to the Informal channel instead, while assuming that the outcomes on the Informal channel remain unchanged. While relevant as a marginal impact, the partial equilibrium result does not take into account all the adjustments that occur in the economy due to the PLX, and therefore is not the appropriate answer for the impact of the PLX for workers and firms.

In the spirit of Heckman, Lochner, and Taber (1998b), I describe how the usual evaluation parameters from a partial equilibrium analysis translate into the PLX general equilibrium framework. The definitions below refer separately to each of the skill-match groups. Usual outcomes in evaluations are wages and unemployment durations.

1. $Y_{1} \mid P L X$ is the treatment outcome conditional on being treated. It is the outcome observed for PLX participants. It can be computed immediately from the general equilibrium model. 
2. $Y_{0} \mid$ Informal is the no treatment outcome conditional on not being treated. It is the outcome observed for participants on the Informal channel. It can be computed immediately from the general equilibrium model.

3. $Y_{0} \mid P L X$ is the no treatment outcome conditional on being treated. It is the counterfactual outcome for PLX participants had they entered the Informal channel instead. In general equilibrium, it can be computed by following PLX participants in a simulation with the PLX channel shut down. In partial equilibrium, it can be computed as the outcome for a similar type agent searching on the Informal channel in the model economy.

4. $Y_{1} \mid$ Informal is the treatment outcome conditional on not being treated. It is the counterfactual outcome for participants on the Informal channel had they entered the PLX channel instead. In general equilibrium, it can be computed following participants on the Informal channel in a simulation with the Informal channel shut down. In partial equilibrium, it can be computed as the outcome for a similar type agent searching on the PLX channel in the model economy.

5. $Y_{1}$ is the average treatment outcome. It is the outcome for searchers on either channel (any random person in the population) had they entered the PLX channel. In general equilibrium, it can be computed as the outcome in a simulation with only the PLX available and the Informal channel shut down. In partial equilibrium, it can be computed as a weighted average of outcomes from the PLX weighted by the skill distribution in the economy.

6. $Y_{0}$ is the no treatment outcome. It is the outcome for searchers on either channel (any random person in the population) had they entered the Informal channel. In general equilibrium, it can be computed as the average outcome in a simulation with only the Informal channel available and the PLX channel shut down. In partial equilibrium, it can be computed as a weighted average of outcomes from the Informal channel weighted by the skill distribution in the economy.

Treatment on the treated (TT) is perhaps the most interesting parameter for evaluators, because it gives the impact of the program for a participant. It measures the effect of a program on a random participant $(\mathrm{PLX}), T T=E\left[\left(Y_{1}-Y_{0}\right) \mid P L X\right]=E\left[Y_{1}\left|P L X-Y_{0}\right| P L X\right]$. This parameter can be computed as the difference in average outcomes for PLX participants between the general equilibrium model (with both channels populated) and the simulation 
where the PLX channel is shut down and everybody is induced to search on the Informal channel. The simulation produces the counterfactual outcome for program participants had the PLX not been available. ${ }^{24}$

Outcomes are computed from the model economy and from the two simulations with each search channel shut down one at a time. Two main categories of parameters can be generated from simulations: a full general equilibrium version of the treatment parameters, where all quantities are allowed to adjust; and a partial equilibrium version where prices (wages in this case) are held fixed.

\section{Calibration}

\subsection{The Definition of Skill}

Workers are separated into two groups, high-skill and low-skill, based on education, experience and wages. The model generates three types of wages: two for high-skill workers - in high-skill and in low-skill jobs - and one for low-skill workers in low-skill jobs. The empirical procedure also has to identify three skill categories: high-skill workers with high wages $w(H, H)$, high-skill workers with low wages $w(H, L)$, and low skill workers who can only earn the low-skill wage $w(L, L)$, where $w(H, H)>w(H, L)>w(L, L)$. In order to identify these three skill groups in the data, the empirical analysis uses the Panel Study of Income Dynamics (PSID) from 1996 to 1999 , and proceeds along the following steps: ${ }^{25}$

(i) Generate the predicted wage from a Mincer wage regression using the PSID data with controls for education categories and for labor market experience. These controls are used since I want to compute the wage thresholds off a smoothened expected wage where the most important predictors are education and experience.

(ii) Investigate the distribution of the predicted wage. A plot from a 50 bin histogram is used and, given how the histogram is shaped, the following quantiles for the wage skill

\footnotetext{
${ }^{24}$ There are other parameters of interest in the evaluation literature. For instance, the Average Treatment Effect (ATE) parameter is defined as the impact of a program on a random individual from the population, $A T E=E\left[Y_{1}-Y_{0}\right]$. In the general equilibrium framework, this would translate into the difference in mean outcomes between the simulation when all agents enter the PLX channel and the simulation when all agents enter the Informal channel. Such a parameter would be of policy relevance if, for instance, policy makers consider making a program mandatory for the entire eligible population.

${ }^{25}$ I thank one of the referees for suggesting such an approach.
} 
distribution are chosen: top 14 bins for the $H H$ workers, middle 8 bins for the $H L$ workers, and bottom 28 for the $L L$ workers. ${ }^{26}$ This boils down to a wage cut-off of $\$ 15.5$ between $L L$ and $H L$ workers and $\$ 18$ between $H L$ and $H H$ workers. The workers are re-classified into the three skill groups, $H H, H L$ and $L L$, depending on where their actual wage falls within the cut-offs. ${ }^{27}$

(iii) Once the new skill distribution has been pinned down as described above, use that information in order to compute various targets in the data that relate to high-skill and low-skill workers and vacancies, as described in greater detail below.

\subsection{Parameters Calibrated Directly from the Data}

Three of the parameters representing exogenous quantities: the fraction of high-skilled labor force $\eta$, and the job destruction rates for high-skill and low-skill, $\delta^{H}$ and $\delta^{L}$, are chosen directly from the data. Three more parameters: the discount factor $\beta$, the bargaining power $s$ and the matching parameter $\alpha$, are taken from the literature. Finally, normalizations are imposed on some search cost parameters. Table 1 reports these calibrated parameters and their values, accompanied by more details on the calibration and data sources.

\subsubsection{The Fraction of High-skill and Low-skill Individuals in the Labor Force}

Given the cut-offs described above, using the 1996-1999 PSID, those who are classified as $H H$ or $H L$ are considered to be high-skill, while those who are classified as $L L$ are considered to be low-skill. This skill definition implies that $45 \%$ of the labor force is high-skilled, i.e. $\eta=0.45$. It is positively, but not perfectly, correlated with education. For instance, $82 \%$ of those with noncollege education are low-skill, while $18 \%$ are classified as high-skill under this skill definition. Likewise, $87 \%$ of the college-educated are classified as high-skill under this skill definition, while $13 \%$ are classified as low-skill. This provides an education-skill conversion matrix for the labor force which will be used later.

\footnotetext{
${ }^{26}$ Similar results can be inferred from histograms with different bandwidths. Moreover, the analysis is for men only, but all the results carry over when women are added to the analysis, with separate conditional wage cut-offs for women. These histograms are available upon request.

${ }^{27}$ For those who are unemployed, their predicted wage is used.
} 


\subsubsection{The Destruction Rate for High-skill and Low-skill Jobs}

Data from the Job Openings and Labor Turnover Survey (JOLTS) is used to compute the destruction rates for high-skill and low-skill jobs. JOLTS reports job destruction rates by onedigit industries. ${ }^{28}$ In order to convert those into high-skill and low-skill job destruction rates, I use the wage data and wage cut-offs from the PSID described above in order to compute the fraction of high-skill and low-skill jobs in each one-digit industry. Unlike for the worker side, where $H L$ matches were classified as high-skill (since those are high-skill workers in low-skill jobs), here the $H L$ matches are counted as low-skill jobs, as it is the skill of the job that is relevant. Therefore, I use (i) the job skill distribution in each one-digit industry, and (ii) the relative size of each one-digit industry, in order to derive the destruction rates for high-skill and low-skill jobs from the information available from JOLTS. This skill definition implies a destruction rate of $10 \%$ for high-skill jobs and $11 \%$ for low-skill jobs, i.e. $\delta^{H}=0.10$ and $\delta^{L}=0.11 .^{29}$

\subsubsection{Parameters Taken from the Literature}

Three more parameters - the discount factor $\beta$, workers' bargaining power $s$, and the matching parameter $\alpha$ - are set to values accepted in the literature. Given the quarterly time frame, the discount factor $\beta$ is calibrated to a $5 \%$ annual interest rate, resulting in $\beta=0.9878$. Workers' bargaining power in the Nash framework is set at $s=0.5$. The matching function used here comes from den Haan, Ramey, and Watson (2000), who calibrate a value of $\alpha=1.27$ for the matching parameter.

\subsubsection{Normalizations and Set Parameters}

The following normalizations are imposed on the parameter space. The productivity of a lowskill job is set to one, $f(L)=1$. Because of this normalization, wages and output are to be interpreted only in a relative way. It is changes in wages and the level of output that are

\footnotetext{
${ }^{28}$ JOLTS is available from December 2000 to July 2003. The separation rates for 2001 - the year used in the computation because it is the closest one to the time-frame here--, are very close to the separation rates for the entire period.

${ }^{29}$ Separation rates are defined as the number of total separations as a percent of total employment. To the extent that these data include separations due to job-to-job transitions (as well as transitions out of the labor force) for which the model does not account, these job destruction rates may overstate the total amount of job destruction.
} 
relevant; the nominal values have no immediate significance per se. There is no loss of generality embedded in this assumption, since the magnitude of general equilibrium adjustments can be quantified from the relative changes in nominal wages and output.

Some restrictions are imposed on the search cost parameters in the model. The benefits of being unemployed, net of search costs, are set equal on the two channels: $(b-c)_{1}=(b-c)_{2}$. Because a worker of given skill level has the same value functions on either channel, and because low-skill workers can only work in low-skill jobs (whose productivity has been normalized to one), this leads to low-skill workers having the same probability of meeting a low-skill vacancy on either channel. ${ }^{30}$ The costs of posting vacancies are imposed to be equal between high-skill and low-skill vacancies on the PLX, as well as equal to the cost of posting a low-skill vacancy on the Informal channel: $k_{2}(H)=k_{2}(L)=\kappa$.

Since only the relative efficiency in matching matters across the two channels, the matching factors of proportionality $\lambda$ are set to 1 on the PLX channel (high and low-skill matching pools) $\lambda_{2 H}=\lambda_{2 L}=1$, while the matching factor of proportionality on the Informal channel, $\lambda_{1}$, is picked up in the calibration. For reasons discussed in the model section, we should expect that $\lambda_{1}>1$, although this restriction is not imposed ex-ante in the calibration.

\subsection{Parameters Calibrated such that Model Moments Match the Data}

Seven parameters remain to be determined. They are: the productivity of a high-skill job $f(H)$, the benefit of unemployed search net of costs $b-c$, the cost of posting a high-skill vacancy on the Informal channel $k_{1}(H)$, all other vacancy posting costs $\kappa$, the probability that PLX misidentifies the skill type of the worker $d_{x}$ or of the job $d_{y}$, and the matching factor of proportionality $\lambda_{1}$.

The parameters are computed in an exactly identified fashion by iterating on the parameter space until seven moments from the model match the same seven targets in the data. These targets are: the fraction of low-skill searchers in the economy $\gamma$, the fraction of low-skill searchers on the PLX $\gamma_{2}$, the fraction of low-skill vacancies on the PLX $\phi_{2}$, the fraction of

\footnotetext{
${ }^{30}$ Furthermore, since stationarity implies that employment numbers are proportional to unemployment numbers with a factor of proportionality that depends on meeting probabilities, low-skill unemployment rates (unemployed divided by employed plus unemployed) must be equal on the two channels.
} 
high-skill to low-skill vacancy fillings on PLX $\frac{e_{2 H H} \cdot \rho_{H}}{\left(e_{2 H L}+e_{2 L L}\right) \cdot \rho_{L}}$, the market tightnesses on both the high-skill and low-skill pools on the PLX $\theta_{2 H}$ and $\theta_{2 L}$, and the unemployment duration $\sum_{j=1,2 x=H, L}\left(\frac{u_{j}(x) / u}{\sum_{y \leq x} p_{j}(x, y)}\right)$. Table 2 gives the model moments used in the calibration, together with the corresponding targets in the data. ${ }^{31}$

\subsubsection{Low-skill Searchers in the Economy}

Given the cut-offs described above, using the 1996-1999 PSID, those unemployed workers who are classified as $H H$ or $H L$ are considered to be high-skill while those who are classified as $L L$ are considered to be low-skill. This skill definition implies that $66 \%$ of the unemployed are low-skill, i.e. $\gamma=0.66$.

\subsubsection{Low-skill Searchers on the PLX}

The U.S Department of Labor (ETA-DOL) provides information about workers who are searching on the PLX; in particular, it reports the fraction of college and non-college searchers on the PLX. I use this information, together with the education-skill conversion matrix for unemployed workers, to compute the fraction of searchers on the PLX who are low-skill. It implies that $75 \%$ of all searchers on the PLX are low-skill: $\gamma_{2}=0.75$. All other targets related to high-skill or low-skill searchers on the PLX are computed in a similar way.

\subsubsection{The Fraction of Low-skill Vacancies on the PLX}

The U.S Department of Labor (ETA-DOL) also provides information on the type of vacancies posted on the PLX; in particular, it reports the number of vacancies (in a given period of time) by one-digit occupations. I use the wage data and wage cut-offs from the PSID described above in order to compute the fraction of high-skill and low-skill jobs in each one-digit occupation. Like for the industries computation, the $H L$ matches are counted as low-skill jobs, as it is the skill of the job that is relevant. Therefore, I use (i) the job skill distribution in each one-digit occupation, and (ii) the relative size of each one-digit occupation, in order to derive the fraction of high-skill and low-skill vacancies from the information available from ETA-DOL. Usually, jobs in Professional and Managerial occupations are considered to be high-skill while jobs in the other one-digit occupations are considered to be low-skill. Such a definition of skill is positively,

\footnotetext{
${ }^{31}$ Computation and data sources are described in the table notes.
} 
but not perfectly, correlated with the one I am using in my analysis. For instance, $70 \%$ of the jobs in the Professional and Managerial occupations are high-skill while 30\% get classified as low-skill. Likewise, $77 \%$ of the jobs in the Non-professional and Non-managerial occupations are classifed as low-skill while $23 \%$ get classified as high-skill.

This skill definition implies that $70 \%$ of the posted vacancies on the PLX are low-skill: $\phi_{2}=0.70$. All other targets related to the high-skill or low-skill vacancies on the PLX are computed in a similar way.

\subsection{Discussion}

The model matches well all of the targets, with the exception perhaps of unemployment duration, where there is a difference of about 0.3 quarters. ${ }^{32}$ The calibrated probabilities that the PLX will make a mistake in identifying workers or vacancies are respectively $4 \%$ and $10.5 \%$. The calibrated matching factor on the Informal channel is $\lambda_{1}=1.431$, which is larger than the factor normalized to one on the PLX, as in order to calibrate an equilibrium with both skill types on both channels, the Informal matching technology must have some attractive feature to balance the sorting provided by the PLX. This attractiveness gets reflected by the higher matching efficiency for the Informal channel, as picked up in the calibration.

Other moments generated from the model, which were not targeted in the calibration, fare reasonably well. In terms of vacancies, the share of low-skill vacancies on the PLX, $\phi_{2}$, was targeted. The low-skill share of vacancies overall was not targeted, nor were vacancies on the Informal channel targeted. Once JOLTS data are combined with ETA-DOL data to get total vacancies (and implicitly vacancies on the Informal channel), the statistics from the data are similar to those computed in the model: $\phi=.617$ in the data and $\phi=.591$ in the model for the share of low-skill aggregate vacancies, and $\phi_{1}=.590$ in the data and $\phi_{1}=.567$ in the model for the share of low-skill Informal sector vacancies. ${ }^{33}$

The model does a good job in replicating other performance statistics reported by the PLX. For instance, the placement rate, reported by the PLX as the fraction of workers entering

\footnotetext{
${ }^{32}$ Data used in the calibration comes from the late 1990 s and early 2000 s, because that was the program year for the PLX statistics. In more recent years, unemployment durations had gone up, so that the duration moment predicted by the model is not larger than later unemployment durations.

${ }^{33}$ Data source: JOLTS 2001 for job openings for the entire economy (channels 1 and 2 together); once weights are applied, there are 17,995,704.86 low-skill and 11,152,295.14 high-skill vacancies (annualized figures) for the economy, while on the PLX the numbers are 5200002.01 and 2261570.99 for low and high-skill respectively.
} 
employment out of those referred, is $52.2 \%$, while the corresponding quantity generated by the calibrated model is only slighly higher, $57.9 \% .^{34}$

The ratio of high-skill to low-skill wages is 1.21 in the model while it is 1.36 in the data. ${ }^{35}$ The model predicts wages $w(H, H)$ which are too low relative to $w(L, L)$, and conversely wages $w(H, L)$ which are too high relative to the low wage. In the model the ratios of $\frac{w(H, H)}{w(L, L)}$ and $\frac{w(H, L)}{w(L, L)}$ are respectively 1.29 and 1.126 , while in the data these are 1.546 and 1.043. In order to match the other targets, the value picked in the calibration for $f(H)=1.27$ seems to be slightly low relative to $f(L)=1$.

The model also has some difficulty in matching the unemployment rates data. In the model the overall unemployment rate is $13.9 \%$. In the data, however, unemployment rates can range from $3.93 \%$ (for men aged 25 or more) up to $6.12 \%$ (for both genders without any age restriction imposed). ${ }^{36}$ Besides the extremely favorable labor circumstances of late 1990s and early 2000s, there are other potential explanations why the model fails to predict lower unemployment rates. One is that the rather high job destruction rates include job to job transitions in the data, which the model does not allow for. Another potential explanation is that only the unemployed are searching in the model. To this extent, unemployment rates reflect the number of job searchers in the economy rather than the genuinely unemployed. The model performs much better in terms of the employment rate, as there is a clear immediate link between employed in the model and employed in the data. In the PSID data the employment rate is $86.37 \%$ for men aged 25 or more, compared to the $86.10 \%$ employment rate predicted from the model.

Finally, Appendix V provides sensitivity results when the calibrated parameters are moved, one at a time, $1 \%$ up and $1 \%$ down around the calibrated equilibrium values. The sensitivity analysis reports changes in the model moments (top panel of Tables A-1 and A-2) as well as changes in the equilibrium impacts of the PLX (bottom panel) from the variation in parameter values. The main findings from the benchmark calibration are robust to changes in the param-

\footnotetext{
${ }^{34}$ Data source for PLX performance indicators: ETA9002-C; 3454759 referred workers entered employment, compared to 6613785 searching on PLX (footnote [2] from Table 2). In the model, in equilibrium the flow of job placements equals the flow of job losses on each channel. Therefore, the placement rate is $\left(e_{2 H H} \delta^{H}+e_{2 H L} \delta^{L}+e_{2 L L} \delta^{L}\right) /\left(u_{2 H}+u_{2 L}\right)$. Employment and unemployment levels from the model are reported in Table 3.

${ }^{35}$ Data source: author's calculations from the 1997 PSID. The corresponding regression-adjusted wage ratio could be as low as 1.234, depending on demographic and job history controls used in the regression. The unadjusted wage gap is higher, up to 1.547.

${ }^{36}$ Data source for unemployment and employment rates: author's calculations from the 1997 PSID.
} 
eters, as increasing or decreasing these parameters has a predictable, but not dramatic, effect on the results.

\section{General and Partial Equilibrium Impacts}

\subsection{Model Economy}

The first column in Table 3 gives the equilibrium outcome in the calibrated model. The next two columns report results from the two experiments when the search channels are shut down one at a time. The adjustments are described in more detail in the next subsection 6.2. Unemployment, employment, and vacancies by skill group and by channel are reported as ratios rather than levels; total quantities remain reported in levels. The Informal channel is more efficient at overall matching $\left(\lambda_{1}=1.431\right.$ relative to the baseline $\left.\lambda_{2}=1\right)$, while the PLX provides a sorting service by skill type, subject to misidentifying worker and vacancy types, with mistake probabilities $d_{w}=4 \%$ in identifying worker types and $d_{v}=10.5 \%$ in identifying job requirements.

A high-skill worker prefers to meet a high-skill vacancy, since the penalty for meeting a lowskill vacancy is loss in output, therefore loss in surplus and wages. For a low-skill worker, to meet a high-skill vacancy has worse consequences because such a meeting results in no productive match being formed. Consequently, the sorting provided by the PLX is very attractive for low-skill workers, and a larger part of the low-skill search and matching takes place on the PLX. The ratio of low-skill to high-skill unemployed is 2.7 on the PLX and 1.8 on the Informal channel. Vacancies follow, with similarly higher ratios of low-to-high skill vacancies: a ratio of 2.2 of low to high vacancies listed on the PLX channel, relative to a ratio of 1.3 on the Informal channel. In the model, the PLX channel is smaller, having a share of about $20 \%$ of the workers' search market (20\% of employed found a jobs on the PLX, while $23 \%$ of the unemployed searched on the PLX), and a share of about $20 \%$ of the total vacancy postings. ${ }^{37}$ The overall unemployment duration is slightly higher on the PLX because it takes longer for high-skill workers to match with a job on the PLX. ${ }^{38}$

\footnotetext{
${ }^{37}$ This is consistent with similar statistcis in the data; on the CPS, about $20 \%$ of workers list the PLX as a job search method, and from the merged JOLTS and PLX statistics, 25\% of the vacancies are listed on the PLX.

${ }^{38}$ The low-skill unemployment durations on the two search channels are the same because low-skill vacancy posting costs and workers' search costs are the same on the two channels.
} 
High-skill workers are more likely to match with a high-skill vacancy when searching on the PLX, which results in a higher fraction of high-skill workers employed in high-skill rather than low-skill jobs, compared to the Informal channel. Subsequently, the high-skill wage on the PLX channel is higher than the high-skill wage on the Informal channel. ${ }^{39}$ Likewise, aggregate average wages on the PLX are lower than on the Informal channel because of the relatively higher fraction of low-skilled searchers on the PLX. For the same reason, that high-skill workers are more likely to get sorted into high-skill jobs on the PLX, labor productivity is higher on the PLX. ${ }^{40}$

Wages and value functions for employed workers indicate, as expected, that high-skill workers in high-skill jobs fare the best, followed by high-skill workers in low-skill jobs, and lastly by low-skill workers. For the firms though, low-skill jobs filled with low-skill workers generate larger surplus than low-skill jobs filled with high-skill workers, because the wages that must be paid to the high-skill workers are higher. The surplus for high-skill workers in low-skill jobs is nevertheless positive, even if small: a high-skill worker maximizing discounted value utility still finds it optimal to accept a lower paid, low-skill job with a shorter expected duration $1 / \delta^{L}$ rather than forgo all surplus until a high-skill match can be established. Likewise for the firms, they prefer to fill a low-skill vacancy with a costlier high-skill worker rather than leave the vacancy empty until a low-skill worker comes along.

\subsection{Experiments}

The second column in Table 3 provides equilibrium quantities from the simulation where the PLX channel is shut down and everybody must search on the Informal channel, which is now the only search channel available. Likewise, the last column in Table 3 has the results from the simulation with the Informal channel shut down, when everyone is searching on the PLX.

\footnotetext{
${ }^{39}$ Wages for high-skill workers in high-skill jobs are the same on the two channels, and likewise wages for highskill workers in low-skill jobs. The difference in the aggregate high-skill wage only comes from the difference in the relative size of the two groups, $H H$ and $H L$, on the two channels.

${ }^{40}$ The aggregate wage is $w=w(H, H) \cdot e_{H H} / e+w(H, L) \cdot e_{H L} / e+w(L, L) \cdot e_{L L} / e$. Total output is $Y=$ $e_{H H} \cdot f(H)+\left(e_{H L}+e_{L}\right) \cdot f(L)$. The corresponding labor productivity measure of output-per-worker is $y=Y / e$. The aggregate value for workers, in some sense a measure of welfare, is $\sum_{x \geq y} W(x, y) \frac{e_{x y}}{e}+\sum_{x} U(x) \frac{u_{x}}{u}$ with $x \in\{H, L\}$ and $y \in\{H, L\}$.
} 


\subsubsection{Experiment 1: Economy without the PLX Channel}

While the Informal channel is more efficient in overall matching, the PLX channel, which used to provide a sorting service, is no longer there. Consequently, more matches are formed but relatively fewer high-skill workers match with high-skill jobs. Because of the higher fraction of high-skill workers in low-skill jobs, the average labor productivity also goes down. The amount of output produced by high-skill workers in high-skill jobs decreases, and the output produced in low-skill jobs increases, because more people are employed, albeit in low-skill jobs. Aggregate output does not increase though, because high-skill workers in low skill jobs, whose relative size has increased, produce less than high-skill workers in high-skill jobs, whose relative size has decreased.

Due to the more efficient matching on the Informal channel, vacancies get filled faster on the average once the PLX is shut down. As a result average unemployment duration goes down, firms fill more vacancies, and employment goes up. While the fraction of low-skill vacancies increases to $64.8 \%$ compared to the baseline case of $59.1 \%$, the number of low-skill vacancies stays roughly the same, and the adjustment comes from a decrease in the total number of high-skill vacancies.

Unemployment incidence and duration also go down, both for high-skill and for low-skill searchers, for several reasons: (i) the Informal channel is more efficient at matching, (ii) highskill workers are less picky about the correct match in the absence of direct sorting, and (iii) there are no more mistakes made in identifying low-skill searchers as high-skill ones.

High-skill workers receive slightly lower wages in the economy without the PLX, whether they work in high-skill jobs or in low-skill jobs, while low-skill workers get a slightly higher wage. The reason is that the economy without PLX operational has fewer high-skill vacancies and relatively more low-skill ones; furthermore, outside options deteriorate for both skill workers because the sorting provided by the PLX is no longer available. For high-skill workers both the vacancies mechanism and the sorting mechanism ensure lower wages in the absence of the PLX; for low-skill workers the mechanism from relatively more low-skill vacancies without the PLX dominates the sorting channel, and as a result low-skill wages are higher without the PLX.

The aggregate wage is lower, because of the larger fraction of high-skill in low-skill jobs. In comparative statics terms, an increase in wages corresponds to a proportional increase in the 
value functions for workers (both employed and unemployed) and a proportional decrease in job values. ${ }^{41}$ As a result of wage adjustments, the value of a high-skill job goes up, as does the value of a low-skill job employing a high-skill worker, while the value of a low-skill job goes down. The opposite adjustment occurs to the values for workers, employed and unemployed: it goes down for the high-skill and up for the low-skill; overall, the values for workers increase. ${ }^{42}$

\subsubsection{Experiment 2: Economy without the Informal Channel}

For equilibrium quantities other than value functions, the reverse adjustments are true if the Informal channel is shut down and everyone has to search on the PLX. There is more sorting going on, because the PLX sorting service is available on the search channel, but overall matching efficiency is lower, $\lambda_{2}=1$. Waiting for the right match takes longer for high-skill workers, who experience longer unemployment duration and incidence. Because of lower matching efficiency, total employment goes down, and the overall number of vacancies increases. Since all searches are directed by PLX employees with relatively low error frequencies, a higher fraction of high-skill workers is employed in high-skill jobs (30\% high-skill workers in high-skill jobs, compared to $23.4 \%$ in the baseline case and $17.4 \%$ in the experiment with no PLX). This leaves fewer high-skill vacancies, while the number of low-skill vacancies increases slightly.

Compared to the baseline economy, unemployment duration is higher (1.665 quarters), but lower than the unemployment duration on the PLX in the baseline case (1.741 quarters). While the high-skill on PLX have the same unemployment duration in the baseline case compared to the experiment without the Informal channel, the low-skill unemployment duration is lower in the experiment, which correlates with a lower fraction of low-skill unemployed competing for the low-skill jobs; $59.6 \%$ compared to $66.4 \%$ (or $72.7 \%$ ) low-skill unemployed in the baseline case, as a share of total unemployed (or share of PLX unemployed).

Due to directed matching, there are fewer poor matches of high-skill workers into low-skill jobs and labor productivity (output per worker) is higher at 1.082 (compared to 1.05 on the

\footnotetext{
${ }^{41}$ The factor of proportionality is $\partial W / \partial w=\frac{1-s(1-\beta) / B}{(1-s)(1-\beta)}$ for the employment value, larger $\partial U / \partial w=\frac{1}{(1-s)(1-\beta)}$ for the unemployment value, and negative $\partial J / \partial w=\frac{-s(1-\beta) / B}{(1-s)(1-\beta)}$ for the value of a job.

${ }^{42}$ Because the bargaining parameter is set at half, $s=0.5$, in equilibrium each party appropriates half of the total surplus, that is, $W(x, y)-U(x)=J(x, y)=S(x, y) / 2$. The changes in value functions are directly related to the changes in wages: when the wages increase, corresponding value functions for workers go up (both the value of work and the outside option) and the value for firms goes down. Because of the Nash rule of splitting the surplus, it must be that changes in the outside option for workers $(U)$ dominate the changes in the work value $(W)$ so the surplus can mimic the value of the job.
} 
baseline case and 1.048 in the case of the experiment without the PLX). The higher productivity also gets reflected in higher total output, which increases despite the reduction in the number of employed workers. Another consequence of the improved assortative matching (fewer high-skill workers in the lower-paying, low-skill jobs) is that the aggregate wage for high-skill workers goes up, even as the wages for high-skill workers in high-skill jobs and in low-skill jobs each go down.

In terms of workers' welfare (as captured by value functions), shutting down the Informal channel results in value functions moving in the same direction as when the PLX was shut down: going down for high-skill workers and up for low-skill workers, only the magnitude of the change is larger for the decreases and smaller for the increases.As a result, on the aggregate value functions for workers decrease. The opposite adjustments hold true for firms' profits, as measured by the job value functions.

\subsection{Program Impact Results}

The implications from Table 3 are reinforced by the program impact analysis, which summarizes some of the equilibrium adjustment and recasts them in the program evaluation terminology. The focus is on the Treatment on the Treated (TT) impact. This is the most often estimated parameter in the evaluation literature, as it answers the very relevant question of what is the impact of the program for its participants. As described in Section 4, to recover the TT only the calibrated model and the first experiment with the PLX channel shut down are needed. The second experiment, with the Informal channel shut down, is relevant for evaluation parameters other than the TT. TT is computed as the difference in outcomes between the calibrated model with agents on the PLX and the outcome from the simulation with the PLX shut down. If wages went up in the experiment without the PLX, this would correspond to a negative impact of the PLX on wages, since workers' wages were higher without the PLX. Likewise, if unemployment durations went down in the experiment without the PLX, this would correspond to a positive impact of the PLX on unemployment durations - only in this case a positive impact implies longer durations in the model economy, generally perceived as undesirable.

Treatment on the treated (TT) impact results are reported for wage outcomes and for unemployment duration outcomes. Table 4 reports the impact of PLX on participants' outcomes; 
participants are individuals who search on the PLX in the baseline model. Table 5 reports the PLX impacts for non-participants, that is, for those individuals who search on the Informal channel in the baseline model. Impacts are reported by skill group and also on the aggregate. The first column gives general equilibrium impacts (discussed first) and the second column gives partial equilibrium impacts (discussed last).

\subsubsection{General Equilibrium Impacts of the PLX}

(i) The impact on participants' wage by skill groups.

From the results in Table 4, it would seem the PLX does not help participants when looking at the aggregate outcomes traditionally considered in evaluation: wages and unemployment durations. Nevertheless, the story is different when skill heterogeneity is taken into account. The TT general equilibrium wage impacts for high-skill workers are positive and reasonably large, at $6.4 \%$. For the low-skill searchers the wage impacts are small and negative: low-skill wages are $1.5 \%$ higher in the economy without the PLX, indicating that, despite the sorting provided by PLX, low-skill workers would actually gain a little in terms of wages if the PLX were not available as a search option. There are two mechanisms, working in the same direction for high-skill workers and in opposite directions for the low-skill workers: (i) all workers have better outside options with the PLX, because of the sorting provided, and (ii) there are more high-skill vacancies when the PLX is available, which is helpful for the high-skill, and detrimental for the low-skill.

\section{(ii) The impact on participants' aggregate wage.}

The impact on aggregate outcomes is not the same as the weighted impact by skill levels, as the weights used in aggregation come from the shares of each skill group.

$$
\Delta w=\left[w(H)_{\text {model }} \frac{e_{H}}{e}{ }_{\text {model }}-w(H)_{\exp } \frac{e_{H}}{e} e_{\text {exp }}\right]+\left[w(L)_{\text {model }} \frac{e_{L}}{e}{ }_{\text {model }}-w(L)_{\text {exp }} \frac{e_{L}}{e} e_{\text {exp }}\right]
$$

where the subscripts model and exp index respectively the model economy and the counterfactual simulation experiment. ${ }^{43}$ As long as the ratio of high-skill to low-skill employed workers changes between the model economy and the experiment, which it does, the impact of PLX

\footnotetext{
${ }^{43}$ The wage impact for high-skill (low-skill) workers is the difference in the high-skill (low-skill) wage between the model economy and the counterfactual experiment, $\Delta w(H)=w(H)_{\text {model }}-w(H)_{\text {exp }}$, and $\Delta w(L)=w(L)_{\text {model }}-w(L)_{\exp }$. The impact for the aggregate wage is:

$\Delta w=w_{\text {model }}-w_{\text {exp }}=\left[w(H)_{\text {model }} \frac{e_{H}}{e}\right.$ model $+w(L)_{\text {model }} \frac{e_{L}}{e}$ model $]-\left[w(H)_{\text {exp }} \frac{e_{H}}{e} \exp +w(L)_{\exp } \frac{e_{L}}{e} \exp \right]$, which leads to 25 .
} 
on the aggregate wage can be very different from the weighted high-skill and low-skill wage impacts; this heterogeneity is a major source of differences between the general and the partial equilibrium impacts of the PLX. In particular, the low-skill wage increases and the high-skill wage decreases in the experiment with the PLX shut down. Since the impacts are computed as baseline wage minus wage in the experiment, the wage impact is negative for the low-skill $(\Delta w(L)<0)$ and positive for the high-skill $(\Delta w(H)>0)$. At the same time, the fraction of employed high-skill workers goes up in the experiment, which can dampen or even negate the contribution of the high-skill wage impact in the aggregation. Furthermore, the high-skill wage impact reported here is itself an aggregation of the wages of high-skill working $\mathrm{n}$ high-skill jobs and wages of high-skill working in low-skill jobs. Movements in the share of these two groups of high-skill workers, which are very pronounced depending on the availability of the PLX sorting, or lack thereof, result in large changes of the high-skill wage impact itself. ${ }^{44}$ Likewise, the increase in the fraction of employed low-skill workers will exacerbates the negative low-skill wage impact in aggregation. In this instance, from the large positive wage impact for the high-skill workers of $6.4 \%$ and the small negative wage impact of $-1.5 \%$ for the low-skill workers, the aggregation produces a very small negative aggregate wage impact of $-0.5 \%$.

\section{(iii) The impact on participants' unemployment duration.}

From the bottom panel of Table 4, the TT general equilibrium duration impacts are positive both for high-skill and for low-skill workers. This means that the individuals searching on the PLX in the model economy would experience a few extra weeks of being unemployed, .456 quarters (almost 6 weeks) for the high-skill searchers and .208 quarters (2.7 weeks) for the low-skill searchers compared to a hypothetical world with the PLX not available. Durations in the presence of the PLX are higher because, for the high-skill workers, it is worth waiting to be sorted into high-skill jobs, while for the low-skill workers the fraction of low-skill vacancies is lower (compared to the experiment with the PLX shut down).

\section{(iv) The impact of the PLX on other outcomes.}

Traditional evaluation outcomes such as wages and unemployment durations do not provide the whole picture. The PLX has a positive impact on labor productivity, which goes down from 1.065 to 1.048 in the economy without the PLX, and on total output, which goes down from

\footnotetext{
${ }^{44}$ While impacts are only reported for the aggregate high-skill wage, separate impacts for high-skill in highand low-skill jobs can always be inferred from the information in Table 3.
} 
9.17 to 9.19 in the economy without the PLX. These are all relevant outcomes to be considered, especially if policy recommendations are supposed to follow the impact analysis of the PLX. ${ }^{45}$

\subsubsection{Partial Equilibrium Impacts of the PLX}

The partial equilibrium impacts are reported in the last column of Tables 4 and 5. Following the assumptions in standard evaluation literature, partial equilibrium impacts are computed from the counterfactual experiment where PLX searchers are assigned to the Informal channel instead, with the number and skill mix of searchers and vacancies fixed on the Informal channel to baseline values. Partial equilibrium adjustments in the experiment with shutting down the PLX channel arise mostly from aggregation, given that prices for a given skill match $(H H, H L, L L)$ do not change. Because low-skill workers can only work in low-skill jobs, there are no aggregation issues regarding the unemployment duration and wages of the low-skilled, and the low-skilled partial equilibrium impacts are zero. For the high-skill, the impacts are an aggregation over the $H H$ and $H L$ wage and duration impacts, and differences can only arise because of the different fractions of $H H$ versus $H L$ on the two channels.

The partial equilibrium aggregate wage impact is positive and $1.6 \%$ of the base wage, compared to a negative impact of $-0.5 \%$ of the base wage for the general equilibrium impact. The partial equilibrium impacts are in line with what the literature has documented: ALMP impacts, including PLX, are generally found to be small, and not always positive. Given the range of impacts in the evaluation literature, we can not rule out that the general equilibrium impacts are conforming to the impacts documented in the literature. Nevertheles, it is notable that in the particular model investigated here, partial and general equilibrium wage impacts have opposing signs.

The partial equilibrium duration impact is 1.6 weeks increase in unemployment duration (0.128 quarters), compared to 4.17 weeks increase in duration (.321 quarters) in the general equilibrium impact. The literature in general finds small but negative impacts on unemployment durations (where a negative impact is a desirable one, as it reduces clients' unemployment durations). In this sense, the partial equilibrium impacts in the model are closer to those

\footnotetext{
${ }^{45}$ Also, while the welfare impacts (value functions) for workers move in the same direction as the wage impacts, the surplus, linearly and positively related to the firm value, moves in the opposite direction. It goes up for firms offering high-skill vacancies, and it goes down for firms offering low-skill vacancies, reflecting the changes in workers' outside options and wages.
} 
documented in the literature, while the general equilibrium ones depart more from the "no impact/small reduction in unemployment weeks" from the literature.

\subsubsection{The Impact of the PLX on Non-participants}

The impacts of the PLX for non-participants are reported in Table 5. These impacts are computed, for agents initially on the Informal channel, as the difference in outcomes between the calibrated model and the simulation with PLX shut down. ${ }^{46}$ In the standard evaluation literature, it is assumed that the outcomes for individuals who do not participate in a program (in our case Informal channel searchers who do not go to the PLX) are not affected by the program being operated, at least not beyond the initial participation decision. This assumption is called "Stable Unit Treatment Value Assumption," or SUTVA. In our case, it imposes the condition that the outcomes of individuals on the Informal channel should be affected neither by the presence of the PLX nor by its absence. This assumption is obviously violated by the very nature of general equilibrium adjustments. Here, by getting a direct measure of the effect of the PLX on non-participants, we can quantify how much we depart from the SUTVA assumption.

Furthermore, note the following relationship by skill level: by construction, the partial equilibrium impacts for participants are the difference between the general equilibrium impacts for participants minus the general equilibrium impacts for non-participants. While this relationship between partial and general equilibrium effects is true by skill level, it is no longer true on aggregate, because the skill composition changes endogenously in general equilibrium. Furthermore, as SUTVA sets by assumption the equilibrium impacts of non-participants to zero, it implicitly also sets the partial equilibrium impacts equal to the general equilibrium ones; a comparison between partial and general equilibrium magnitudes is equivalent to an assessment of whether SUTVA holds or not.

One very important result here is that the general equilibrium impacts of the PLX on nonparticipants, assumed to be zero under SUTVA, are of similar magnitude with the impacts for participants: $1.1 \%$ increase in wages, and 1.6 weeks (.129 quarters) increase in unemployment duration.

\footnotetext{
${ }^{46}$ This should not be confused with another parameter popular in the evaluation literature, the Treatment on the Not Treated (TNT) parameter. TNT is a different parameter which can be recovered as the difference in outcomes between an experiment where the Informal channel is shut down (forcing Informal searchers to go to the PLX) and the model economy.
} 
Finally, all the results show remarkable heterogeneity by skill group. The high-skill workers experience different outcomes compared to the low-skilled ones. Furthermore, there are also significant changes within the high-skill outcomes themselves, due to equilibrium changes in the fractions of high-skill workers working in high-skill jobs versus low-skill jobs.

\section{Conclusion and Extensions}

This paper extends the framework introduced by Albrecht and Vroman (2002), where firms decide endogenously to create high-skill and low-skill jobs, by introducing explicitly two search channels in the model. One is a standard random-matching Mortenssen-Pissarides type of channel, while the other channel has a directed search component to the extent that PLX employers perform a screening of worker and vacancy types and facilitate the meeting among "right" types of workers and vacancies. On the theoretical side, the approach modeled here can be a useful tool for thinking about directed search in a different way than for instance recent approaches by Shi (2002) or Gonzalez and Shi (2010).

This framework is a very useful tool for quantifying general equilibrium effects in the evaluation of the PLX program, and also for comparing general with partial equilibrium results that are the status-quo in the current evaluation literature. General equilibrium adjustments come from several sources: the congestion of searchers on different channels, employers altering the number and skill mix of the vacancies opened in the presence of the PLX, changes in the matching opportunities and outside options for workers, and different quality of matches. An economy without the PLX is more efficient at overall matching but less efficient at sorting worker and job skills. Consequently, if the PLX is no longer available, high-skill workers fare worse because a larger fraction of them end up working in low-skill jobs. At the same time, low-skill workers fare better because there are more overall matches happening, and a higher fraction of low-skill vacancies. In the absence of PLX sorting, unemployment durations decrease because matching on the Informal channel is more efficient. Labor productivity also decreases because of the lower match quality.

One insight yielded from these results is that researchers should use caution when interpreting estimation results obtained in a partial equilibrium framework. If the program operates on a large enough scale, as is the case of the PLX, then it is likely that the standard program eval- 
uation assumption of no general equilibrium effects would be violated. Once adjustments by other actors in the economy are considered, the impact of a program can be very different, even opposite at times from the partial equilibrium one. Moreover, while wages and unemployment durations remain relevant outcomes, they are not the only ones affected. In the particular case of the PLX, productivity adjustments are similarly important, and policy inference based on counterfactuals from traditional partial equilibrium estimates may not tell the whole story.

There are several avenues that would merit further investigation. An experiment which would shut down the PLX only for low-skill workers, prohibiting them from searching on the PLX (while leaving this channel open for high-skill searchers) would give the counterfactual outcome for low-skill searchers on the PLX had the PLX not been available. Such an experiment would produce the impact of the PLX for low-skill workers, who are the primary clients of the PLX. The model developed here should also be a very useful framework for examining policy implications such as mandating PLX participation for all workers and firms. Additionally, it could be useful to see how a change in the PLX matching technology (such as a reduction in the misidentification probabilities) would impact on the economy. Moreover, further research could expand the current framework to incorporate training programs that affect human capital accumulation. 


\section{References}

Adison, J., And P. Portugal (2002): "Job Search Methods and Outcomes," Oxford Economic Papers, (54), 505-533.

Albrecht, J., G. van den Berg, and S. Vroman (2005): "The Knowledge Lift: The Swedish Adult Education Program That Aimed to Eliminate Low Worker Skill Levels," IZA Discussion Paper, (1503).

Albrecht, J., and S. VRoman (2002): "A Matching Model with Endogenous Skill Requirements," International Economic Review, 43(1), 283-305.

Anderson, P. (2001): "Monitoring and Assisting Job Search," in Labour Market Policies and the Public Employment Service. OECD.

Bishop, J. (1993): "Improving Matches in the Labor Market," Brookings Papers on Economic Activity, 1, 335-400.

Blundel, R., M. C. Dias, C. Meghir, and J. van Reenen (2004): "Evaluating the Employment Impact of a Mandatory Job Search Program," Journal of the European Economic Association, 2(4), 569-606.

Boone, J., And J. C. van Ours (2004): "Effective Active Labor Market Policies," Discussion Paper 1335, Institute for the Study of Labor (IZA).

Burdett, K., And E. Cunningham (1998): "Toward a Theory of Vacancies," Journal of Labor Economics, 16(3), 445-478.

Cohen, M., And D. Stevens (1989): "The Role of the Employment Service," In Investing in People, Background Papers, vol.1, Final Report of the Commission on Workforce Quality and Labor Market Efficiency, U.S. Department of Labor, pp. 1027-1069.

Davidson, C., And S. Woodbury (1993): "The displacement effects of re-employment bonus programs," Journal of Labor Economics, 11(4), 575-605. 
(2000): "Crowding-out effects of the Public Labor Exchange in Washington State," In "Measuring the effect of Public Labor Exchange referrals and placements in Washington and Oregon" by Louis Jacobson and Ian Petta, Westat.

den Haan, W. J., G. Ramey, and J. Watson (2000): "Job Destruction and Propagation of Shocks," The American Economic Review, 90(3), 482-498.

Fougere, D., J. Pradel, And M. Roger (2006): "Does Job-Search Assistance Affect Search Effort and Outcomes? A Microeconometric Analysis of Public versus Private Search Methods," Mimeo.

Gonzalez, F., And S. Shi (2010): “An Equilibrium Theory of Learning, Search and Wages," Econometrica, 78, 509-537.

GregG, P., And J. Wadsworth (1996): "How Effective are State Employment Agencies? Jobcentre Use and Job Matching in Britain," Oxford Bulletin of Economics and Statistics, 58(3), 443-468.

Heckman, J., L. Lochner, and C. Taber (1998): "Explaining Rising Wage Inequality: Explorations with a Dynamic General Equilibrium Model of Labor Earnings with Heterogeneous Agents," Review of Economic Dynamics, 1, 1-58.

— (1998b): "General Equilibrium Treatment Effects: A Study of Tuition Policy," American Economic Review, Papers and Proceedings, 88(2), 381-386.

Jacobson, L., and I. Petta (2000): "Measuring the Effect of Public Labor Exchange (PLX) Referrals and Placements in Washington and Oregon," Discussion paper, Westat Inc. under contract for U.S. Department of Labor, Washington DC.

Johnson, T., K. Dickinson, And R. West (1985): "An Evaluation of the Impact of Employment Service Referrals on Applicant Earnings," The Journal of Human Resources, 20(1), 117-137.

Katz, A. (1991): "The Length of Joblessness and the Employment Service with Special Reference to Philadelphia and Pittsburgh, Pennsylvania, 1979-1987," in The Potential Effectiveness of the Employment Service in Serving Dislocated Workers under EDWAA: Evidence 
from the 1980s, ed. by D. C. Carol J. Romero, and A. Katz. Washington, DC: National Commission for Employment Policy.

Lindeboom, M., J. van Ours, and G. Renes (1994): "Matching Employed Workers: an Empirical Analysis on the Effectiveness of Search," Oxford Economics Papers, 46(1), 46-67.

Lise, J., S. Seitz, And J. Smith (2004): "Equilibrium Policy Experiments and the Evaluation of Social Programs," Working paper 10283, NBER.

OECD (2001): "Labour Market Policies and the Public Employment Service," Discussion paper.

O'LeARy, C. J. (2004): "Effectiveness of Labor Exchange Services," in "Labor Exchange Services in the United States: History, Effectiveness, and Prospects" David E. Balducchi, Randall W. Eberts and Christopher J. O'Leary, eds. Upjohn Institute for Employment Research.

Osberg, L. (1993): "Fishing in Different Pools: Job Search Strategies and Job-Finding Success in Canada in the early 1980s," Journal of Labor Economics, 11(2), 348-86.

Petrongolo, B., and C. Pissarides (2001): "Looking Into the Black Box: a Survey of the Matching Function," Journal of Economic Literature, 39(2), 390-431.

Roper, S. (1988): "Recruitment Methods and Vacancy Duration," Scottish Journal of Political Economy, 35(1), 51-64.

SHI, S. (2002): "A Directed Search Model of Inequality with Heterogeneous Skills and SkillBiased Technology," Review of Economic Studies, 69, 467-491.

VAN OuRs, J. (1993): "Matching the Unemployed and Vacancies at the Public Employment Office," Empirical Economics, 19, 37-54.

VAn Ours, J., And G. Ridder (1993): "Vacancy Durations: Search or Selection," Oxford Bulletin of Economics and Statistics, 55(2), 189-98.

Wolpin, K., And P. Todd (2006): "Using Experimental Data to Validate a Dynamic Behavioral Model of Child Schooling: Assessing the Impact of a School Subsidy Program in Mexico," American Economic Review, 96(5), 1384-1417. 
Table 1: Calibrated Parameters

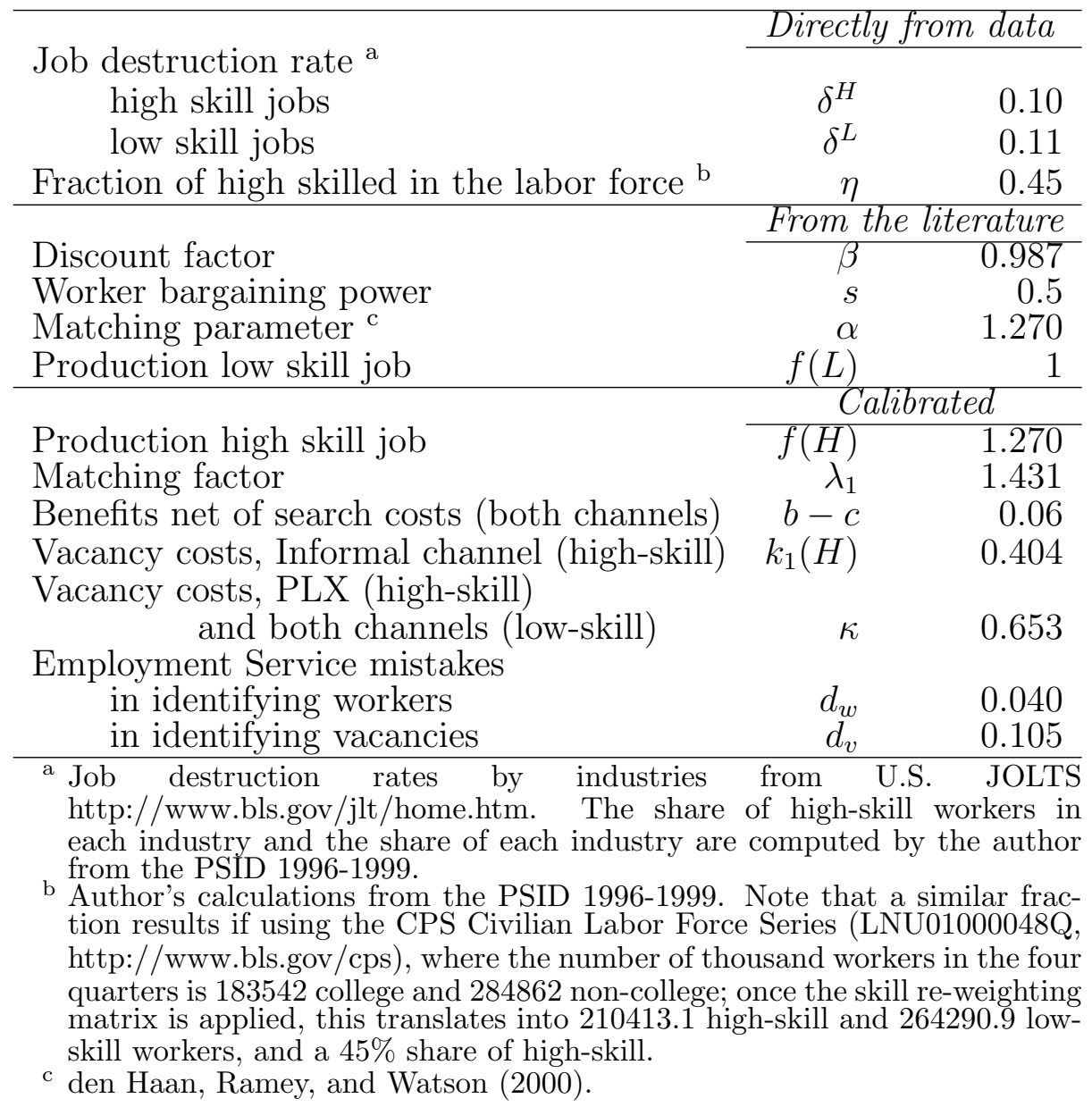


Table 2: Model Moments and Data Targets

\begin{tabular}{llllc}
\hline \multicolumn{1}{c}{ Moment } & Definition & Model & Data $^{*}$ \\
\hline 1 & Low skill searchers in the economy & $\gamma=u_{L} / u$ & 0.66 & 0.66 \\
2 & Low skill searchers on PLX & $\gamma_{2}=u_{2 L} / u_{2}$ & 0.73 & 0.75 \\
3 & Fraction low skill vacancies on PLX & $\phi_{2}=v_{2 L} / v_{2}$ & 0.70 & 0.70 \\
& Fraction high-skill to low-skill vacancy fillings on PLX & $\frac{e_{2 H} \cdot \rho_{H}}{\left(e_{2 H}+e_{2 L}\right) \cdot \rho_{L}}$ & 0.32 & 0.36 \\
5 & Market tightness on the high skill pool on PLX & $\theta_{2 H}$ & 1.36 & 1.36 \\
6 & Market tightness on the low skill pool on PLX & $\theta_{2 L}$ & 1.05 & 1.05 \\
7 & Unemployment duration & $\sum \frac{u_{j}(x) / u}{\sum_{y \leq x} p_{j}(x, y)}$ & 1.59 & 1.23 \\
\hline
\end{tabular}

Author's calculations using the following data sources:

(Weighting of workers from education to skill and of vacancies from occupation to skill is done using weights computed by the author from PSID; see Section 5.1).

1 PSID 1996-1999.

2 ETA9002-C. 6,613,785 worker referrals by the PLX, out of which 5,843,559 non-college and 770,226 college. Applying worker skill conversion weights (author's calculation, PSID) results in 4957461.312 low-skill and 1656323.688 high-skill referrals. http://www.doleta.gov

3 ETA9002-C. 7,461,573 vacancy openings received by the PLX, of which 1,120,430 professional, technical or managerial occupations. Applying worker skill weights (author's calculation, PSID) results in 5,200,002 low-skill and 2,261,571 high-skill vacancies.

4 ETA9002-C. 1,756,264 non-professional and non-managerial vacancies filled by the PLX, and 136,235 professional, technical and managerial vacancies filled by PLX. Applying occupation skill conversion weights (author's calculation, PSID) results in 1,445,881.7 low-skill and 446,617.3 high-skill occupations filled by the PLX.

${ }^{5}$ High-skill vacancies over high-skill worker referrals. See notes 2 and 3.

${ }_{7}^{6}$ Low-skill vacancies over low-skill worker referrals. See notes 2 and 3.

7 U.S. Bureau Of Labor Statistics, CPS Unemployment duration 1999. Data converted from months to quarters (1 quarter $=13$ weeks).

http://www.bls.gov/cps 
Table 3: Experiments

\begin{tabular}{|c|c|c|c|}
\hline & $\begin{array}{c}\text { Model } \\
\text { Economy }\end{array}$ & $\begin{array}{l}\text { Without } \\
\text { PLX }\end{array}$ & $\begin{array}{l}\text { Without } \\
\text { Informal }\end{array}$ \\
\hline \multicolumn{4}{|l|}{ Unemployment ratio } \\
\hline$u_{1 H} / u$ & 0.275 & 0.352 & \\
\hline$u_{1 L} / u$ & 0.502 & 0.648 & \\
\hline$u_{2 H} / u$ & 0.061 & & 0.404 \\
\hline$u_{2 L} / u$ & 0.163 & & 0.596 \\
\hline$u$ & 0.139 & 0.128 & 0.150 \\
\hline \multicolumn{4}{|l|}{ Employment ratio } \\
\hline$e_{1 H H} / e$ & 0.184 & 0.174 & \\
\hline$e_{1 H L} / e$ & 0.220 & 0.291 & \\
\hline$e_{1 L L} / e$ & 0.401 & 0.536 & \\
\hline$e_{2 H H} / e$ & 0.050 & & 0.295 \\
\hline$e_{2 H L} / e$ & 0.014 & & 0.163 \\
\hline$e_{2 L L} / e$ & 0.130 & & 0.542 \\
\hline \multirow{2}{*}{\multicolumn{4}{|c|}{ Vacancy ratio }} \\
\hline & & & \\
\hline$v_{1 H} / v$ & 0.350 & 0.352 & \\
\hline$v_{1 L} / v$ & 0.459 & 0.648 & \\
\hline$v_{2 H} / v$ & 0.059 & & 0.175 \\
\hline$v_{2 L} / v$ & 0.132 & & 0.825 \\
\hline & 0.186 & 0.174 & 0.167 \\
\hline \multicolumn{4}{|l|}{ Unemployment durations } \\
\hline duration $1 H$ & 1.037 & 1.050 & \\
\hline duration $1 L$ & 1.828 & 1.621 & \\
\hline duration $_{1}$ & 1.548 & & \\
\hline duration $2 H$ & 1.507 & & 1.506 \\
\hline duration $2 L$ & 1.828 & & 1.773 \\
\hline duration ${ }_{2}$ & 1.741 & & \\
\hline duration & 1.591 & 1.420 & 1.665 \\
\hline \multicolumn{4}{|l|}{ Wages } \\
\hline$w(H, H)$ & 1.092 & 1.081 & 1.076 \\
\hline$w(H, L)$ & 0.953 & 0.942 & 0.937 \\
\hline$w(H)$ & 1.023 & 0.994 & 1.027 \\
\hline$w(L)$ & 0.846 & 0.859 & 0.850 \\
\hline$w$ & 0.929 & 0.922 & 0.931 \\
\hline \multicolumn{4}{|l|}{ Output } \\
\hline$Y_{1}(H)$ & 0.203 & 0.193 & \\
\hline$Y_{1}(L)$ & 0.535 & 0.720 & \\
\hline$Y_{2}(H)$ & 0.055 & & 0.320 \\
\hline$Y_{2}(L)^{\prime}$ & 0.124 & & 0.599 \\
\hline$Y$ & 0.917 & 0.914 & 0.919 \\
\hline \multicolumn{4}{|l|}{ Output-per-worker (productivity) } \\
\hline$y_{1}=Y_{1} / e_{1}$ & 1.064 & & \\
\hline$y_{2}=Y_{2} / e_{2}$ & 1.072 & & \\
\hline$y=Y / e$ & 1.065 & 1.048 & 1.082 \\
\hline \multicolumn{4}{|l|}{ Surplus } \\
\hline$S(H, H)=J(H, H) /(1-s)$ & 3.353 & 3.548 & 3.635 \\
\hline$S(H, L)=J(H, L) /(1-s)$ & 0.778 & 0.958 & 1.037 \\
\hline$S(L)=J(L, L) /(1-s)$ & 2.544 & 2.337 & 2.490 \\
\hline overall & 2.321 & 2.146 & 2.591 \\
\hline
\end{tabular}


Table 3: Experiments (continued)

\begin{tabular}{|c|c|c|c|}
\hline & $\begin{array}{c}\text { Model } \\
\text { Economy }\end{array}$ & $\begin{array}{l}\text { Without } \\
\text { PLX }\end{array}$ & $\begin{array}{l}\text { Without } \\
\text { Informal }\end{array}$ \\
\hline \multicolumn{4}{|l|}{ Value of employment for workers } \\
\hline$W(H, H)$ & 75.933 & 74.254 & 73.510 \\
\hline$W(H, L)$ & 74.645 & 72.959 & 72.211 \\
\hline$W(L, L)$ & 58.035 & 59.982 & 58.542 \\
\hline overall & 66.116 & 66.234 & 65.189 \\
\hline \multicolumn{4}{|l|}{ Value of unemployment } \\
\hline$U(H)$ & 74.256 & 72.480 & 71.693 \\
\hline$U(L)$ & 56.763 & 58.814 & 57.297 \\
\hline overall & 62.639 & 63.623 & 63.106 \\
\hline \multicolumn{4}{|l|}{ Aggregate value for workers } \\
\hline$\sum_{x \geq y} W(x, y) \frac{e_{x y}}{e+u}+\sum_{x} U(x) \frac{u_{x}}{e+u}$ & 65.634 & 65.899 & 64.875 \\
\hline
\end{tabular}

Table 4: Treatment on the Treated (TT) Wage and Unemployment Duration Impacts for PLX participants

\begin{tabular}{ccc}
\hline TT Wage impacts & General eq. & Partial eq. \\
\hline$w$ impact & -0.004 & 0.015 \\
$\%$ of base wage & $-0.5 \%$ & $1.6 \%$ \\
$w(H)$ impact & 0.068 & 0.045 \\
$\%$ of base wage & $6.4 \%$ & $4.3 \%$ \\
$w(L)$ impact & -0.013 & 0 \\
$\%$ of base wage & $-1.5 \%$ & $0 \%$ \\
\hline Duration impacts (quarter) & General eq. & Partial eq. \\
\hline TT duration impact & 0.321 & 0.128 \\
duration impact for high-skill & 0.456 & 0.469 \\
duration impact for low-skill & 0.208 & 0 \\
\hline
\end{tabular}

Table 5: Wage and Unemployment Duration Impacts for PLX nonparticipants

\begin{tabular}{ccc}
\hline Wage impacts & General eq. & Partial eq. \\
\hline$w$ impact & 0.01 & \\
$\%$ of base wage & $1.1 \%$ & zero \\
$w(H)$ impact & 0.022 & under \\
$\%$ of base wage & $2.2 \%$ & SUTVA $^{\text {a }}$ \\
$w(L)$ impact & -0.013 & \\
$\%$ of base wage & $-1.5 \%$ & \\
\hline Duration impacts (quarter) & General eq. & Partial eq. \\
\hline Non-participant duration impact & 0.129 & zero \\
duration impact for high-skill & -0.013 & under \\
duration impact for low-skill & 0.208 & SUTVA $^{\text {a }}$ \\
\hline a Note: Stable Unit Treatment Value Assumption (SUTVA) is the standard \\
assumption of no general equilibrium effects, routinely employed in the eval- \\
uation literature.
\end{tabular}




\section{APPENDICES}

\section{Matching Probabilities on the PLX Channel}

1. Effective market tightness for high-skill agents

$$
\widetilde{\theta}_{2, H}=\frac{\widetilde{v}_{2, H}}{\widetilde{u}_{2, H}}=\frac{\left(1-d_{v}\right) v_{2, H}+d_{v} v_{2, L}}{\left(1-d_{w}\right) u_{2, H}+d_{w} u_{2, L}}=\frac{v_{2}}{u_{2}}\left[\frac{\left(1-d_{v}\right)\left(1-\phi_{2}\right)+d_{v} \phi_{2}}{\left(1-d_{w}\right)\left(1-\gamma_{2}\right)+d_{w} \gamma_{2}}\right] .
$$

2. Effective market tightness for low-skill agents

$$
\widetilde{\theta}_{2, L}=\frac{\widetilde{v}_{2, L}}{\widetilde{u}_{2, L}}=\frac{d_{v} v_{2, H}+\left(1-d_{v}\right) v_{2, L}}{d_{w} u_{2, H}+\left(1-d_{w}\right) u_{2, L}}=\frac{v_{2}}{u_{2}}\left[\frac{d_{v}\left(1-\phi_{2}\right)+\left(1-d_{v}\right) \phi_{2}}{d_{w}\left(1-\gamma_{2}\right)+\left(1-d_{w}\right) \gamma_{2}}\right] .
$$

3. Some simplifying notation: Denote at this point by $\Psi$ and by $\Gamma$ the following quantities: ${ }^{47}$

$$
\begin{aligned}
& \Psi=\left(1-d_{v}\right)\left(1-\phi_{2}\right)+d_{v} \phi_{2}, \\
& \Gamma=\left(1-d_{w}\right)\left(1-\gamma_{2}\right)+d_{w} \gamma_{2} .
\end{aligned}
$$

The formulas for effective market tightness can be written as:

$$
\begin{aligned}
& \widetilde{\theta}_{2, H}=\theta_{2} \frac{\Psi}{\Gamma} \\
& \widetilde{\theta}_{2, L}=\theta_{2} \frac{(1-\Psi)}{(1-\Gamma)} .
\end{aligned}
$$

With this notation, the meeting functions on the PLX channel become: ${ }^{48}$

$$
\begin{gathered}
M_{2, H}=\widetilde{u}_{2, H} \cdot \widetilde{m}_{2, H} \text {; and } \\
M_{2, L}=\widetilde{u}_{2, L} \cdot \widetilde{m}_{2, L} \text {, where } \\
\widetilde{m}_{2, H}=\frac{\widetilde{\theta}_{2, H}}{\left(1+\widetilde{\theta}_{2, H}^{\alpha}\right)^{\frac{1}{\alpha}}}=\frac{\Psi \theta_{2}}{\left[\Gamma^{\alpha}+\Psi^{\alpha} \cdot \theta_{2}^{\alpha}\right]^{\frac{1}{\alpha}}} ; \text { and } \\
\widetilde{m}_{2, L}=\frac{(1-\Psi) \theta_{2}}{\left(1+\widetilde{\theta}_{2, L}{ }^{\alpha}\right)^{\frac{1}{\alpha}}}=\frac{\left.(1-\Gamma)^{\alpha}+(1-\Psi)^{\alpha} \cdot \theta_{2}^{\alpha}\right]^{\frac{1}{\alpha}}}{[(1-}
\end{gathered}
$$

\footnotetext{
${ }^{47}$ Note that $\Psi$ and $\Gamma$ are linear equations of $\phi$ and $\gamma$ respectively. If $d_{v}=0$, then $\Psi=1-\phi$.

${ }^{48}$ The factor of proportionality $\lambda_{2}$ is normalized to $\lambda_{2}=1$ for the PLX, and therefore is omitted from these formulas.
} 
Using the $\Psi$ and $\Gamma$ simplified notation, the following quantities can be computed:

$$
\begin{aligned}
& \frac{v_{2, H}}{\widetilde{v}_{2, H}}=\frac{1-\phi_{2}}{\Psi} ; \quad \frac{v_{2, H}}{\widetilde{v}_{2, L}}=\frac{1-\phi_{2}}{1-\Psi} ; \quad \frac{v_{2, L}}{\widetilde{v}_{2, H}}=\frac{\phi_{2}}{\Psi} ; \quad \frac{v_{2, L}}{\widetilde{v}_{2, L}}=\frac{\phi_{2}}{1-\Psi} ; \\
& \frac{u_{2, H}}{\widetilde{u}_{2, H}}=\frac{1-\gamma_{2}}{\Gamma} ; \quad \frac{u_{2, H}}{\widetilde{u}_{2, L}}=\frac{1-\gamma_{2}}{1-\Gamma} ; \quad \frac{u_{2, L}}{\widetilde{u}_{2, H}}=\frac{\gamma_{2}}{\Gamma} ; \quad \frac{u_{2, L}}{\widetilde{u}_{2, L}}=\frac{\gamma_{2}}{1-\Gamma} .
\end{aligned}
$$

Therefore, the matching probabilities on the PLX channel can be re-written as:

$$
\begin{aligned}
p_{2}(H, H) & =\left[\frac{\left(1-d_{w}\right)\left(1-d_{v}\right)}{\Psi} \widetilde{m}_{2 H}+\frac{d_{w} d_{v}}{1-\Psi} \widetilde{m}_{2 L}\right]\left(1-\phi_{2}\right), \\
p_{2}(H, L) & =\left[\frac{\left(1-d_{w}\right) d_{v}}{\Psi} \widetilde{m}_{2 H}+\frac{d_{w}\left(1-d_{v}\right)}{1-\Psi} \widetilde{m}_{2 L}\right] \phi_{2}, \\
p_{2}(L, L) & =\left[\frac{d_{w} d_{v}}{\Psi} \widetilde{m}_{2 H}+\frac{\left(1-d_{w}\right)\left(1-d_{v}\right)}{1-\Psi} \widetilde{m}_{2 L}\right] \phi_{2}, \\
q_{2}(H, H) & =\left[\frac{\left(1-d_{w}\right)\left(1-d_{v}\right)}{\Psi} \frac{\widetilde{m}_{2 H}}{\theta_{2 H}}+\frac{d_{w} d_{v}}{1-\Psi} \frac{\widetilde{m}_{2 L}}{\theta_{2 L}}\right]\left(1-\gamma_{2}\right), \\
q_{2}(H, L) & =\left[\frac{\left(1-d_{w}\right) d_{v}}{\Psi} \frac{\widetilde{m}_{2 H}}{\theta_{2 H}}+\frac{d_{w}\left(1-d_{v}\right)}{1-\Psi} \frac{\widetilde{m}_{2 L}}{\theta_{2 L}}\right]\left(1-\gamma_{2}\right), \\
q_{2}(L, L) & =\left[\frac{d_{w} d_{v}}{\Psi} \frac{\widetilde{m}_{2 H}}{\theta_{2 H}}+\frac{\left(1-d_{w}\right)\left(1-d_{v}\right)}{1-\Psi} \frac{\widetilde{m}_{2 L}}{\theta_{2 L}}\right] \gamma_{2} .
\end{aligned}
$$




\section{Solving for the Equilibrium when Both Skill Types Search on Both Channels ("Full-mixing" Equilibrium)}

High-skill workers produce in high- or low-skill jobs; low-skill workers only produce in lowskill jobs. Workers are indifferent between searching on the Informal channel or on the PLX channel, $U_{1}(x)=U_{2}(x)$ for $x=\{H, L\}$. Likewise firms are indifferent where to post vacancies $V_{1}(y)=V_{2}(y)$ for $y=\{H, L\}$. Six unknowns need to be solved for in this equilibrium: $\theta_{j}$, $\gamma_{j}$, and $\phi_{j}$, with $j=\{1,2\}$. The overall quantities of interest can be computed as weighted averages of their respective values on each channel. The market tightness in the economy is $\theta=$ $\theta_{1} \frac{u_{1}}{u_{1}+u P}+\theta_{2} \frac{u P}{u_{1}+u P}$. The fraction of low-skill searchers in the economy is $\gamma=\gamma_{1} \frac{u_{1}}{u_{1}+u P}+\gamma_{2} \frac{u P}{u_{1}+u P}$. The fraction of low-skill vacancies in the economy is $\phi=\phi_{1} \frac{\theta_{1}}{\theta} \frac{u_{1}}{u}+\phi_{2} \frac{\theta_{2}}{\theta} \frac{u P}{u}$.

The following steps give the equilibrium solution. First, re-write (24) for $\{y=H\}$ and $\{y=L\}$, respectively.

$$
\begin{gathered}
(1-\beta) U(H)=f(H)-\frac{B^{H}}{\beta(1-s)} \frac{k_{j}(H)}{q_{j}(H, H)} \quad j=\{1,2\} \\
(1-\beta) U(L)=f(L)-[f(H)-f(L)] \frac{q_{j}(H, L)}{q_{j}(L, L)}+\frac{1}{\beta(1-s)}\left[\frac{B^{H} k_{j}(H)}{q_{j}(H, H)} \frac{q_{j}(H, L)}{q_{j}(L, L)}-\frac{B^{L} k_{j}(L)}{q_{j}(L, L)}\right] \\
j=\{1,2\}
\end{gathered}
$$

1. Eliminate $U(H)$ between the Informal channel equations.

Using equations (23) with $\{j=1\},\{x=H\}$ and (A7) with $\{j=1\}$ gives the equilibrium condition for a high-skill worker to engage in a productive match on the Informal channel:

$$
k_{1}(H)=\frac{q_{1}(H, H)}{B^{H}} \beta(1-s) \frac{\left[f(H)-\left(b-c_{1}\right)\right]+\beta s \frac{p_{1}(H, L)}{B^{L}}[f(H)-f(L)]}{1+\beta s\left[\frac{p_{1}(H, H)}{B^{H}}+\frac{p_{1}(H, L)}{B^{L}}\right]}
$$

2. Eliminate $U(H)$ between the PLX channel equations.

Using equations (23) with $\{j=2\},\{x=H\}$ and (A7) with $\{j=2\}$ gives the equilibrium condition for a high-skill worker to engage in a productive match on the PLX channel:

$$
k_{2}(H)=\frac{q_{2}(H, H)}{B^{H}} \beta(1-s) \frac{\left[f(H)-\left(b-c_{2}\right)\right]+\beta s \frac{p_{2}(H, L)}{B^{L}}[f(H)-f(L)]}{1+\beta s\left[\frac{p_{2}(H, H)}{B^{H}}+\frac{p_{2}(H, L)}{B^{L}}\right]}
$$


3. Eliminate $U(L)$ between the Informal channel equations.

Using equations (23) with $\{j=1\},\{x=L\}$ and $A 8$ with $\{j=1\}$ gives the equilibrium condition for a low-skill worker to engage in a productive match on the Informal channel:

$$
k_{1}(H)-k_{1}(L)=q_{1}(H, L)[f(H)-f(L)] \frac{\beta(1-s)}{B^{L}}-q_{1}(L, L) \frac{f(L)-\left(b-c_{1}\right)}{B^{L}+\beta s p_{1}(L, L)} \beta(1-s)
$$

4. Eliminate $U(L)$ between the PLX channel equations.

Using equations (23) with $\{j=2\},\{x=L\}$ and (A8) with $\{j=2\}$ gives the equilibrium condition for a low-skill worker to engage in a productive match on the PLX channel:

$$
\begin{gathered}
k_{2}(H) \frac{q_{2}(H, L)}{q_{2}(H, H)}-k_{2}(L)= \\
q_{2}(H, L)[f(H)-f(L)] \frac{\beta(1-s)}{B^{L}}-q_{2}(L, L) \frac{f(L)-\left(b-c_{2}\right)}{B^{L}+\beta s p_{2}(L, L)} \beta(1-s)
\end{gathered}
$$

5. Eliminate $U(H)$ between equations for the Informal and PLX channels.

Using equations (A7) with $\{j=1\}$ and (A7) with $\{j=2\}$ gives the equilibrium condition for a high-skill worker to be indifferent between searching on the Informal channel or the PLX: ${ }^{49}$

$$
\frac{k_{1}(H)}{q_{1}(H, H)}=\frac{k_{2}(H)}{q_{2}(H, H)}
$$

6. Eliminate $U(L)$ between equations for the Informal and PLX channels.

Using equations (23) with $\{j=1\},\{x=L\}$ and (23) with $\{j=2\},\{x=L\}$ gives the equilibrium condition for a low-skill worker to be indifferent between searching on the Informal channel or the PLX: ${ }^{50}$

$$
\frac{B^{L}\left(b-c_{1}\right)+\beta s p_{1}(L, L) f(L)}{B^{L}+\beta s p_{1}(L, L)}=\frac{B^{L}\left(b-c_{2}\right)+\beta s p_{2}(L, L) f(L)}{B^{L}+\beta s p_{2}(L, L)}
$$

Equations (A9) to (A14) give the solution for the full-mixing equilibrium.

\footnotetext{
${ }^{49}$ Note that in the special case when $k_{1}(H)=k_{2}(H)=k(H)$, the full-mixing equilibrium is satisfied iff the probabilities that a high skill worker meets a high-skill vacancy are the same on the two channels, i.e. $q_{1}(H, H)=q_{2}(H, H)$.

${ }^{50}$ To capture the fact that high-skill workers and low-skill workers are indifferent between searching on either channel, the complementary set of equations could have been used instead: (23) with $\{j=1\},\{x=H\}$ and (23) with $\{j=2\},\{x=H\}$ for high-skill and (A8) with $\{j=1\}$ and (A8) with $\{j=2\}$ for low-skill. The results should obviously be the same.
} 


\section{II.1 Other Quantities: Computing Employment, Wages and Output from the Equilibrium Solution}

The flow equilibrium conditions (6) to (8) do not yield any relationship between $\gamma$ and $\phi$ in this mixing equilibrium solution. They nevertheless give solutions for unemployment on the two channels. Using the following identities:

$$
u_{j H}=\left(1-\gamma_{j}\right) u_{j} \quad \text { and } \quad u_{j L}=\gamma_{j} u_{j} \quad j=\{1,2\}
$$

and the equilibrium flow equations, one can obtain the measures of employment on each channel, $e_{1}=e_{1 H}+e_{1 L}$ and $e_{2}=e_{2 H}+e_{2, L}$ :

$$
\delta e_{j}=\left\{\left(1-\gamma_{j}\right)\left[p_{j}(H, H)+p_{j}(H, L)\right]+\gamma_{j} p_{j}(L, L)\right\} u_{j} \quad \text { for } j=\{1,2\}
$$

In the cross-skill equilibrium, not all of the employed high-skill workers work in high-skill jobs. In this case,

$$
\delta e_{j H y}=p_{j}(H, y) u_{j H} \quad \text { for } j=\{1,2\}, \quad y=\{H, L\}
$$

gives the measure of high-skill workers employed in, respectively, high skill $y=H$ or low-skill $y=L$ jobs.

Once the equilibrium quantities $\theta, \gamma$ and $\phi$ have been computed, as well as the implied meeting probabilities, then (A15) and (A16) provide the solution for the employment and unemployment equilibrium quantities. Vacancies are also immediate to compute, as $v=\theta u$.

Wages can be solved for using (21) and (23). ${ }^{51}$

$$
\begin{aligned}
& f(y)-w(x, y)=(1-s)\left\{f(y)-\frac{\left(b-c_{j}\right)+\beta s \sum_{y \leq x} \frac{p_{j}(x, y)}{B^{Y}} f(y)}{1+\beta s \sum_{y \leq x} \frac{p_{j}(x, y)}{B^{Y}}}\right\} \\
& \text { for } x=\{H, L\}, \quad y=\{H, L\}, \quad x \geq y
\end{aligned}
$$

Note that $f(H)>f(L) \Rightarrow w(H, H)>w(H, L) .{ }^{52}$

The wage of low-skill workers simplifies to:

$$
f(L)-w(L, L)=(1-s) \frac{B\left[f(L)-\left(b-c_{j}\right)\right]}{B+\beta s p_{j}(L, L)} .
$$

\footnotetext{
${ }^{51}$ Equations (A7) and (A8) could have been used instead of (23) to compute equilibrium wages.

${ }^{52}$ In general, it is difficult to say if $w(H, L)>w(L, L)$ or even if $w(H, H)>w(L, L)$, unless the meeting probabilities are clearly better for high-skill workers. The exception is the corner separating equilibrium with all agents on the Informal channel, where it can be unambiguously shown that $w(H, H)>w(H, L)>w(L, L)$.
} 
Total output in the economy is given by:

$$
Y=e_{H} \cdot f(H)+e_{L} \cdot f(L)=\sum_{j=\{1,2\}} e_{j H H} \cdot f(H)+\left(e_{j H H}+e_{j L}\right) \cdot f(L),
$$

where employment formulas follow from (A16) and (A17).

Start from (7) and (8):

$$
\begin{array}{r}
e_{1 H}+e_{2 H}+u_{1 H}+u_{2 H}=\eta \\
e_{1 L}+e_{2 L}+u_{1 L}+u_{2 L}=1-\eta
\end{array}
$$

Substitute for $e_{1 x}+e_{P x}$ from summing equation (6) when $\{j=1\}$ and when $\{j=2\}$, separately for $x=H$ and $x=L$, to get expressions for unemployment.

$$
\begin{aligned}
& u_{1}=\frac{\delta \eta \gamma_{2}\left(p_{2}(L L)+\delta\right)-\delta(1-\eta)\left(1-\gamma_{2}\right)\left(p_{2}(H H)+p_{2}(H L)+\delta\right)}{\left(1-\gamma_{1}\right) \gamma_{2}\left(p_{1}(H H)+p_{1}(L H)+\delta\right)\left(p_{2}(L L)+\delta\right)-\gamma_{1}\left(1-\gamma_{2}\right)\left(p_{1}(L L)+\delta\right)\left(p_{2}(H H)+p_{2}(H L)+\delta\right)} \\
& u_{2}=\frac{\delta \eta \gamma_{1}\left(p_{1}(L L)+\delta\right)-\delta(1-\eta)\left(1-\gamma_{1}\right)\left(p_{1}(H H)+p_{1}(L H)+\delta\right)}{\left(1-\gamma_{2}\right) \gamma_{1}\left(p_{2}(H H)+p_{2}(H L)+\delta\right)\left(p_{1}(L L)+\delta\right)-\gamma_{2}\left(1-\gamma_{1}\right)\left(p_{2}(L L)+\delta\right)\left(p_{1}(H H)+p_{1}(L H)+\delta\right)}
\end{aligned}
$$

The inequalities that need to be satisfied in this case come from the condition that engaging in a productive match upon meeting is worthwhile, $f(y)>w(x, y)$. Note from (21) and $f(H)>f(L)$ that if the condition for a high-skill worker to take a low-skill job is satisfied, it implies that the high-skill worker would take a high-skill job as well. Therefore, there are only two relevant conditions: $f(L)>w(H, L)$ and $f(L)>w(L, L)$.

On the Informal channel, the two inequalities can be written as:

$$
\begin{gathered}
f(L)>w(H, L) \Leftrightarrow B^{L}\left[f(L)-\left(b-c_{1}\right)\right]-\beta s m\left(\theta_{1}\right)\left(1-\phi_{1}\right)[f(H)-f(L)]>0 \\
f(L)>w(L, L) \Leftrightarrow B^{L}\left[f(L)-\left(b-c_{1}\right)\right]>0
\end{gathered}
$$

On the PLX channel, the two inequalities can be written as:

$$
\begin{gathered}
f(L)>w(H, L) \Leftrightarrow B^{H}\left[f(L)-\left(b-c_{2}\right)\right]>\beta s p_{2}(H, H)[f(H)-f(L)] \\
f(L)>w(L, L) \Leftrightarrow f(L)-\left(b-c_{2}\right)>0
\end{gathered}
$$

By assumption, $f(H)>f(L)$. Therefore, $(A 19) \Rightarrow(A 20)$, and thus (A20) is not binding. Likewise, $(A 22) \Rightarrow(A 21)$, and thus (A21) is not binding. This result is consistent with the stronger statement that $w(H, L)>w(L, L)$, although to show that the wage inequality $w(H, H)>w(H, L)>w(L, L)$ holds in all cases extra assumptions are needed. ${ }^{53}$ Therefore, (A19) and (A22) are the relevant inequalities in the full-mixing equilibrium.

\footnotetext{
${ }^{53}$ The only statement that can be made unambiguously in this case is that $w(H, H)>w(H, L)$. Without
} 


\section{Solving for the Corner Solution when All Agents Mi- grate to One Channel ("Separating" Equilibrium)}

The solution for the separating equilibria is reciprocally the same, regardless whether workers migrate to the Informal or to the PLX channels.

\section{Segregating on the Informal Channel.}

All workers find it optimal to migrate to the Informal channel, $U_{1}(x)>U_{2}(x)$, for $x=\{H, L\}$. Vacancies follow. There are no workers or vacancies on the PLX channel. Subscript "inf" denotes the entire population of searchers and vacancies, $u_{1}=u$ and $v_{1}=v$. There are three unknown quantities that this equilibrium has to solve for: $\theta_{1}, \gamma_{1}$, and $\phi_{1}$.

\section{Segregating on the PLX Channel.}

All workers and vacancies migrate to the PLX channel, $U_{1}(x)<U_{2}(x) \quad$ for $\quad x=\{H, L\}$ Subscript " $p l x$ " denotes the entire population of searchers and vacancies, $u_{2}=u$ and $v_{2}=v$. There are three unknown quantities that this equilibrium has to solve for: $\theta_{2}, \gamma_{2}$, and $\phi_{2}$.

Two of the equilibrium equations come from the conditions that high-skill and low-skill workers engage in productive matches. Therefore, for the Informal channel these are equations (A9) and (A11) derived for the mixing equilibrium. For the PLX channel, they are equations (A10) and (A12). The third equation in solving the separating equilibrium comes from equilibrium flow equations. From (7) and (8) we have that $e_{H}+u_{H}=\eta$ and $e_{L}+u_{L}=1-\eta$. Substitute these back into (6) to get:

$$
\begin{aligned}
& u_{H}=u_{j H}=\frac{\eta}{1+\frac{p_{j}(H, H)}{\delta^{H}}+\frac{p_{j}(H, L)}{\delta^{L}}} \\
& u_{L}=u_{j L}=\frac{1-\eta}{1+\frac{p_{j}(L, L)}{\delta^{L}}} \quad \text { for } j=\{1,2\}
\end{aligned}
$$

further assumptions, the possibility that $w(H, L)<w(L, L)$ cannot be ruled out. The model has different implications from those of Albrecht and Vroman's (2000). In their case, $w(H, H)>w(H, L)>w(L, L)$ unambiguously and the model can explain "between" and "within" wage inequality. A side product of my model is a weakening of the result in Albrecht and Vroman, in the sense that extra assumptions are needed for $w(H, H)>w(H, L)>w(L, L)$ to hold in all cases. 
From $\gamma_{j}=\frac{u_{L}}{u_{H}+u_{L}}$, the following relationship between $\gamma_{j}$ and $\phi_{j}$ results:

$$
\gamma_{j}=\frac{u_{j L}}{u_{j H}+u_{j L}}=\frac{\frac{1-\eta}{1+\frac{p_{j}(L, L)}{\delta^{L}}}}{\frac{\eta}{1+\frac{p_{j}(H, H)}{\delta^{H}}+\frac{p_{j}(H, L)}{\delta^{L}}}+\frac{1-\eta}{1+\frac{p_{j}(L, L)}{\delta^{L}}}} \quad \text { for } j=\{1,2\}
$$

(A9), (A11), and (A23) give the solution for $\theta_{1}$ and for $\gamma_{1}=\gamma_{1}\left(\theta_{1}\right)$ and $\phi_{1}=\phi_{1}\left(\theta_{1}\right)$. (A10), (A12), and (A23) give the solution for $\theta_{2}$ and for $\gamma_{2}=\gamma_{2}\left(\theta_{2}\right)$ and $\phi_{2}=\phi_{2}\left(\theta_{2}\right)$.

There are five inequalities that the parameters need satisfy in the separating equilibrium. The first three inequalities say that the value of a productive match on the PLX channel is higher than the outside options, $f(y)>w(x, y)$. As in the mixing equilibrium, this leads to one inequality only (A22) that needs to be satisfied.

The next two inequalities follow from:

$$
\begin{gathered}
U(x)=U_{1}(x)>U_{2}(x) \Leftrightarrow \\
\sum_{y \leq x}\left[p_{1}(x, y)-p_{2}(x, y)\right][f(y)-w(x, y)] \geq\left(c_{1}-c_{2}\right) \frac{(1-s)}{s} \frac{B}{\beta} \quad \text { for } x=\{H, L\}
\end{gathered}
$$

with $x=H$ and $x=L$. Inequalities A24 have to be in reverse order for the model to be consistent with the PLX channel being always chosen over the Informal channel.

High-skill workers choose the PLX channel $\{j=2\}$ over the Informal channel $\{i=1\}$ if

$$
\begin{gathered}
\frac{p_{j}(H, H)}{B^{H}} f(H)+\frac{p_{j}(H, L)}{B^{L}} f(L) \geq\left(b-c_{j}\right)\left(\frac{p_{j}(H, H)}{B^{L}}+\frac{p_{j}(H, L)}{B^{L}}\right)+ \\
\left(c_{j}-c_{i}\right)\left(\frac{1}{\beta s}+\frac{p_{j}(H, H)}{B^{L}}+\frac{p_{j}(H, L)}{B^{L}}\right) .
\end{gathered}
$$

Low-skill workers choose the PLX channel if

$$
\frac{p_{j}(L, L)}{B^{L}} f(L) \geq\left(b-c_{j}\right) \frac{p_{j}(L, L)}{B^{L}}+\left(c_{j}-c_{i}\right)\left(\frac{1}{\beta s}+\frac{p_{j}(L, L)}{B^{L}}\right) .
$$

Note that, since they depend on equilibrium results, it is possible that inequalities (A25) and (A26) are satisfied both when all agents migrate to the Informal channel and when they migrate to the PLX channel. Moreover, depending on the sign of $c_{2}-c_{1}$, only one of these inequalities will be binding. The inequalities that have to be satisfied for a separating equilibrium to hold are therefore (A22), (A25), and (A26). 


\section{Solving for the Corner Solution when High-skill Work- ers Migrate to the Informal Channel and Low-skill Workers Migrate to the PLX Channel ("Partial-mixing" Equilibrium)}

In this equilibrium, all high-skill workers find it optimal to migrate to the Informal channel, while all low-skill workers search for jobs on the PLX channel, $U_{1}(H)>U_{2}(H)$, and $U_{1}(L)<$ $U_{2}(L)$. Therefore, $u_{1, L}=u_{2, H}=0$. It follows that $\gamma_{1}=0$ and $\gamma_{2}=1$. Moreover, $u_{2, H}=0 \Rightarrow$ $q_{2}(H, H)=q_{2}(H, L)=0$ and therefore $v_{2, H}=0$ and $\phi_{2}=1$. Thus, only three unknowns still need to be determined in this equilibrium: $\theta_{1}, \theta_{2}$, and $\phi_{1}$.

From equation $(24)$, noting that $q_{1}(L, L)=0$ and $q_{1}(H, H)=\frac{m\left(\theta_{1}\right)}{\theta_{1}}$, one can get:

$$
f(H)-f(L)=\left[k_{1}(H)-k_{1}(L)\right] \frac{B^{H}}{\beta(1-s)} \frac{\theta_{1}}{m\left(\theta_{1}\right)} .
$$

Equation (A27) gives an analytical solution for $\theta_{1}$. Note that in order for this equilibrium to be feasible, it must be that $k_{1}(H)>k_{1}(L)$. The second equation comes from (A9). Replacing the formulas for the probabilities $q_{1}(x, y)(\mathrm{A} 9)$ can be rewritten as:

$$
\begin{aligned}
& k_{1}(H) \frac{B^{H}}{\beta(1-s)}\left[B^{H}+\beta s m\left(\theta_{1}\right)\right] \frac{\theta_{1}}{m\left(\theta_{1}\right)}=B^{H}\left[f(H)-\left(b-c_{1}\right)\right]+\beta s m\left(\theta_{1}\right)[f(H)-f(L)] \phi_{1} \\
& \text { or, } \left.\phi_{1}=\frac{B^{H}}{\beta s m\left(\theta_{1}\right)[(f(H)-f(L)]}\left\{k_{1}(H) \frac{B^{H}+\beta s m\left(\theta_{1}\right)}{\beta(1-s)} \frac{\theta_{1}}{m\left(\theta_{1}\right)}-\left[f(H)-\left(b-c_{1}\right)\right]\right\} \quad \text { (A2 }\right)
\end{aligned}
$$

Equation (A12) with $q_{2}(H, L)=$ is used in obtaining the solution for the last unknown, $\phi_{2}$ :

$$
\frac{k_{2}(L)}{\beta(1-s)\left[f(L)-\left(b-c_{2}\right)\right]}=\frac{q_{2}(L, L)}{B^{L}+\beta s p_{2}(L, L)},
$$

where $\phi_{2}$ enters via $p_{2}(L, L)$ and $q_{2}(L, L)$. Therefore, equations (A27), (A28) and (A29) finish the solution for this equilibrium.

The relevant inequalities that need to be satisfied in this equilibrium derive from the following conditions: $f(H)>w(H, H)$ and $f(L)>w(H, L)$ on the Informal channel (the first is not binding, the second is inequality (A19)); $f(L)>w(L, L)$ on the PLX channel (inequality (A21)); high-skill workers choose the Informal channel (inequality (A25) with a reversed sign); and finally low-skill workers choose the PLX channel (inequality (A26)). 


\section{Sensitivity analysis}

Tables A-1 and A-2 report the sensitivity of equilibrium moments, as well as the sensitivity of general and partial equilibrium impacts, to small changes of parameters around their equilibrium values. In particular, each parameter is (i) increased and then (ii) decreased by $1 \%$ around its calibrated value. Table A-1 reports quantity adjustmenst from chaning the calibrated parameters $d x, d y, f(H), b-c_{1}=b-c_{2}, k_{1 H}$ and $k_{1 L}=k_{2 H}=k_{2 L}$; Table A-2 reports sensitivity for $\lambda_{1}, \beta, s, \alpha_{1}$, eta, and $r h o^{H}$ and $r h o^{L}{ }^{54}$

The top panel of each table reports sensitivity results for the equilibrium moments. The "Baseline" column gives the equilibrium moments originally reported in Table 2 in the text. The bottom panel has sensitivity results for the general and partial equilibrium impacts on wages and unemployment duration. The "Baseline" column present the general equilibrium impacts and partial equilibrium impacts initially reported in Tables 4 and 5 in the text.

While the equilibrium moments from the model change very little with the $1 \%$ change in parameter values, in all instances the changes go inthe right direction direction: if a moment goes up when a parameter increases by $1 \%$ then the same moment goes down when that parameter decreses by $1 \%$ - and vice-versa, if the moment goes down when a parameter increases, it goes up when the parameter decreases. The parameters which result in the largest changes are $\lambda_{1}$, the matching efficiency of channel 1 , and the discount factor $\beta$ (which was calibrated to a $5 \%$ interest rate). Their marginal effect on model moments is the opposite: for instance, when $\lambda_{1}$ goes up by 1\%, unemployment duration and high-to-low skill placement rates increase, while all other moments used in calibration (share of low-skill unemployed in the economy and on the PLX, share of low-skill vacancies on the PLX, and the two market tghtness on the PLX) decrease; the opposite adjustments occur from a $1 \%$ increase in $\beta$. The effect from a $1 \%$ change in the high-skill job productivity $f(H)$ is also substantive. The effect from a $1 \%$ change in the other parameters is either small or very small, but nevertheless the changes go in the right direction: if they go up (down) when a parameter is increased, they go down (up) when that parameter is decreased.

The same holds true for the sensitivity of general and partial equilibrium impacts to param-

\footnotetext{
${ }^{54}$ In the last set of parameters, in calibration $s$ and $\alpha_{1}$ were set to values from the literature and $e t a, r h o^{H}$ and $r h o^{L}$ were calibrated directly from the data.
} 
eter changes of $1 \%$ around equilibrium values. Most of the changes in impacts are small, with the exception of the sensitivity on $\lambda_{1}, \beta, f(H)$, and $\kappa$, when the change in impacts is larger. In all cases, nevertheless, the changes go in the expected direction: if an impact increases (decreases) when a parameter goes up, the same impact decreases (increases) when that parameter goes down. 
Table A-1: Sensitivity to $1 \%$ change (increase and decrease) in parameters

\begin{tabular}{|c|c|c|c|c|c|c|c|c|c|c|c|c|c|}
\hline \multirow[b]{2}{*}{ Main moments } & BASELINE & \multicolumn{2}{|c|}{$d x$} & \multicolumn{2}{|c|}{$d y$} & \multicolumn{2}{|c|}{$f(H)$} & \multicolumn{2}{|c|}{$b-c$} & \multicolumn{2}{|c|}{$k_{1 H}$} & \multicolumn{2}{|c|}{$k_{1 L}=k_{2 H}=k_{2 L}$} \\
\hline & 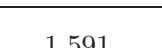 & up $_{159}$ & down & $u_{1594}$ & down & $u_{\mathrm{p}}$ & down & $\mathrm{up}_{\mathrm{s}}$ & down & up & down & up & down \\
\hline $\begin{array}{l}\text { unemp. duur. } \\
\gamma=\underline{u_{L}}\end{array}$ & $\begin{array}{l}1.591 \\
0.664\end{array}$ & $\begin{array}{l}1.592 \\
0.664\end{array}$ & 1.590 & $\begin{array}{l}1.594 \\
0.664\end{array}$ & $\begin{array}{l}1.588 \\
0.664\end{array}$ & $\begin{array}{l}1.576 \\
0.668\end{array}$ & $\begin{array}{l}1.619 \\
0656\end{array}$ & $\begin{array}{l}1.592 \\
0664\end{array}$ & 1.590 & 1.591 & 1.591 & 1.603 & 1.574 \\
\hline$\gamma_{0}=\frac{u_{2 L}^{u}}{u}$ & 0.727 & $\begin{array}{l}0.004 \\
0.726\end{array}$ & $\begin{array}{l}0.004 \\
0.728\end{array}$ & 0.724 & 0.730 & $\begin{array}{l}0.000 \\
0.753 \\
-10\end{array}$ & $\begin{array}{l}0.656 \\
0.691\end{array}$ & $\begin{array}{l}0.064 \\
0.727\end{array}$ & 0.664 & 0.663 & 0.665 & 0.664 & 0.664 \\
\hline$\Phi_{2}^{u_{2}}$ & 0.693 & 0.691 & 0.694 & 0.688 & 0.696 & 0.719 & 0.053 & 0.193 & 0.127 & 0.129 & $\begin{array}{l}0.120 \\
0.689\end{array}$ & $\begin{array}{l}0.121 \\
0.684\end{array}$ & $\begin{array}{l}0.130 \\
0.703\end{array}$ \\
\hline$\theta_{2 H}$ & 1.365 & 1.364 & 1.366 & 1.365 & 1.365 & 1.386 & 1.343 & 1.365 & 1.365 & 1.365 & $\begin{array}{l}.005 \\
1.365\end{array}$ & 1.0049 & $\begin{array}{l}0.103 \\
1.390\end{array}$ \\
\hline$\theta_{2 L}$ & 1.050 & 1.051 & 1.049 & 1.050 & 1.050 & 1.044 & 1.057 & 1.050 & 1.050 & $\begin{array}{l}1.049 \\
1.049\end{array}$ & 1.051 & 1.038 & $\begin{array}{l}1.390 \\
1.067\end{array}$ \\
\hline Plcmt.rt. H/L & 0.314 & 0.316 & 0.312 & 0.321 & 0.309 & 0.269 & 0.387 & 0.316 & 0.315 & 0.311 & 0.32 & 0.328 & 0.297 \\
\hline \multicolumn{14}{|l|}{ Wage impacts: ge } \\
\hline $\begin{array}{l}\text { TTw } \\
\text { TT wH }\end{array}$ & $\begin{array}{c}-0.005 \\
0.067\end{array}$ & $\begin{array}{c}-0.004 \\
0.068\end{array}$ & $\begin{array}{l}-0.005 \\
0.067\end{array}$ & $\begin{array}{c}-0.004 \\
0.068\end{array}$ & $\begin{array}{l}-0.005 \\
0.067\end{array}$ & $\begin{array}{c}-0.011 \\
0.067\end{array}$ & $\begin{array}{l}0.004 \\
0.068\end{array}$ & $\begin{array}{c}-0.004 \\
0.068\end{array}$ & $\begin{array}{l}-0.004 \\
0.067\end{array}$ & $\begin{array}{l}-0.005 \\
0.067\end{array}$ & $\begin{array}{c}-0.004 \\
0.068\end{array}$ & $\begin{array}{c}-0.003 \\
0.069\end{array}$ & -0.007 \\
\hline TT wL & -0.012 & -0.013 & -0.012 & -0.013 & -0.012 & -0.012 & -0.013 & -0.013 & -0.012 & -0.012 & -0.013 & -0.013 & $\begin{array}{r}-0.012 \\
-0.00\end{array}$ \\
\hline non-p w & 0.010 & 0.010 & 0.010 & 0.010 & 0.010 & 0.009 & 0.012 & 0.010 & 0.010 & 0.010 & 0.010 & 0.010 & 0.010 \\
\hline non-p wH & 0.022 & 0.022 & 0.022 & 0.022 & 0.022 & 0.022 & 0.022 & 0.022 & 0.022 & 0.022 & 0.023 & 0.023 & 0.021 \\
\hline \multicolumn{14}{|l|}{$\begin{array}{l}\text { non-p wL }=\mathrm{TT} w \mathrm{w} \\
\text { Wage impacts: pe }\end{array}$} \\
\hline TT w & 0.015 & 0.015 & 0.015 & 0.015 & 0.015 & 0.013 & 0.017 & 0.015 & 0.015 & 0.015 & 0.015 & 0.015 & 0.014 \\
\hline TTwH & 0.045 & 0.045 & 0.045 & 0.045 & 0.045 & 0.045 & 0.046 & 0.045 & 0.045 & 0.045 & 0.045 & 0.046 & 0.045 \\
\hline \multicolumn{14}{|l|}{ Duration impacts: ge } \\
\hline TT dur & 0.3205 & 0.3210 & 0.3200 & 0.3216 & 0.3196 & 0.3204 & 0.3213 & 0.3206 & 0.3208 & 0.3188 & 0.3219 & 0.3252 & 0.3136 \\
\hline TT dur H & 0.4563 & 0.4570 & 0.4557 & 0.4564 & 0.4563 & 0.4475 & 0.4659 & 0.4564 & 0.4564 & 0.4555 & 0.4573 & 0.4599 & 0.4510 \\
\hline TT dur L & 0.2075 & 0.2084 & 0.2066 & 0.2106 & 0.2048 & 0.1994 & 0.2213 & 0.2080 & 0.2080 & 0.2046 & 0.2103 & 0.2154 & 0.1968 \\
\hline non-p dur & 0.1283 & 0.1287 & 0.1279 & 0.1298 & 0.1269 & 0.1303 & 0.1289 & 0.1287 & 0.1287 & 0.1245 & 0.1319 & 0.1343 & 0.1199 \\
\hline \multirow{3}{*}{$\begin{array}{l}\text { Duration impacts: pe } \\
\text { TT dur } \\
\text { TT dur H }\end{array}$} & -0.0127 & -0.0127 & -0.0127 & -0.0129 & -0.0126 & -0.0123 & -0.0135 & -0.0128 & -0.0126 & -0.0124 & -0.0131 & -0.0136 & -0.0117 \\
\hline & 0.1279 & 0.1285 & 0.1273 & 0.1297 & 0.1264 & 0.1138 & 0.1482 & 0.1281 & 0.1279 & 0.1268 & 0.1296 & 0.1323 & 0.1223 \\
\hline & 0.4690 & 0.4696 & 0.4684 & 0.4693 & 0.4689 & 0.4599 & 0.4794 & 0.4690 & 0.4690 & 0.4679 & 0.4704 & 0.4735 & 0.4627 \\
\hline
\end{tabular}


Table A-2: Sensitivity to 1\% change (increase and decrease) in paraneters

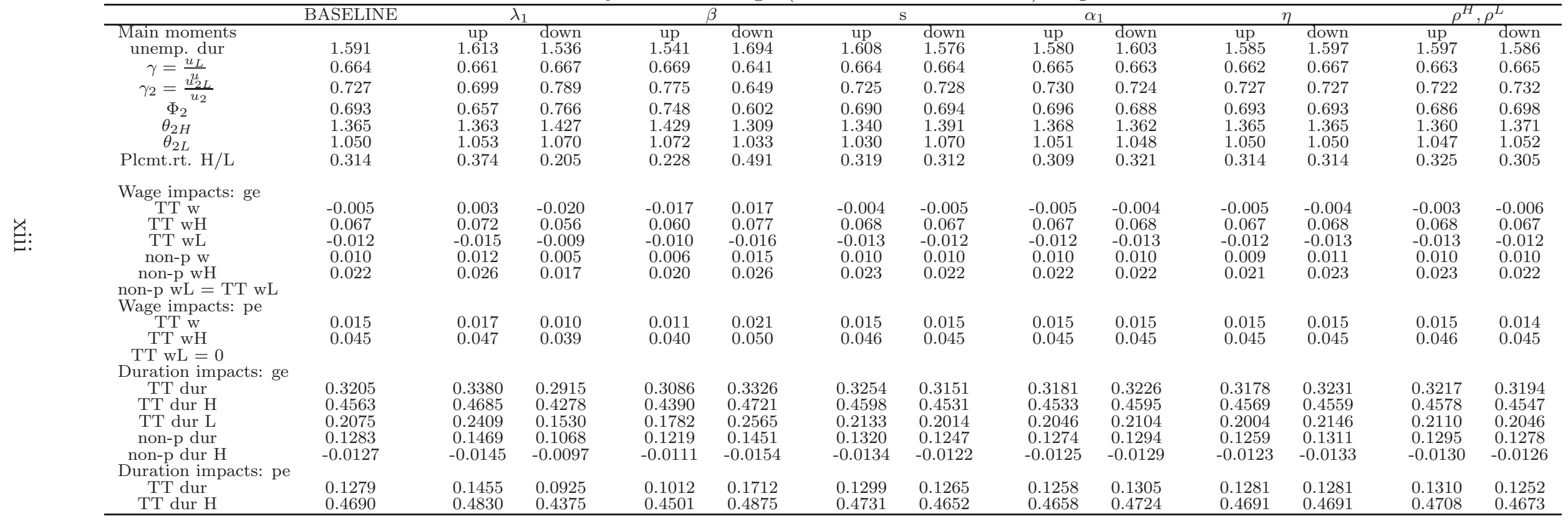

